\title{
Exact correlation functions in the Brownian Loop Soup
}

\author{
Federico Camia, ${ }^{a, b}$ Valentino F. Foit, ${ }^{c}$ Alberto Gandolfi ${ }^{a}$ and Matthew Kleban ${ }^{c}$ \\ ${ }^{a}$ Science Division, NYU Abu Dhabi, \\ Saadiyat Campus, Abu Dhabi, U.A.E. \\ ${ }^{b}$ Department of Mathematics, VU University, \\ De Boelelaan 1081a, 1081 HV Amsterdam, The Netherlands \\ ${ }^{c}$ Center for Cosmology and Particle Physics, New York University, \\ 726 Broadway, New York, NY 10003, U.S.A. \\ E-mail: federico.camia@nyu.edu, foit@nyu.edu, albertogandolfi@nyu.edu, \\ kleban@nyu.edu
}

ABSTRACT: We compute analytically and in closed form the four-point correlation function in the plane, and the two-point correlation function in the upper half-plane, of layering vertex operators in the two dimensional conformally invariant system known as the Brownian Loop Soup. These correlation functions depend on multiple continuous parameters: the insertion points of the operators, the intensity of the soup, and the charges of the operators. In the case of the four-point function there is non-trivial dependence on five continuous parameters: the cross-ratio, the intensity, and three real charges. The four-point function is crossing symmetric. We analyze its conformal block expansion and discover a previously unknown set of new conformal primary operators.

Keywords: Conformal Field Theory, Integrable Field Theories, Random Systems, Stochastic Processes

ArXIV EPRINT: 1912.00973 


\section{Contents}

1 Introduction 1

2 Summary and results $\quad 4$

2.1 Motivation and previous work 5

3 The two-point function in the upper half-plane $\quad 6$

4 The two- and three-point functions in the full plane $\quad 8$

5 The general four-point function in the plane 9

$\begin{array}{lll}5.1 & \text { Free-field limit } & 12\end{array}$

6 Expansion in conformal blocks $\quad \mathbf{1 3}$

$\begin{array}{lll}6.1 \text { Primary operator spectrum } & 15\end{array}$

$\begin{array}{lll}6.2 \text { Interpretation } & 18\end{array}$

$\begin{array}{ll}6.3 & \text { Null descendant states } \\ \end{array}$

$\begin{array}{llr}7 \text { Outlook } & 19\end{array}$

$\begin{array}{ll}\text { A Full-plane limits } & 19\end{array}$

B Weights of loops covering one point and not a second 23

\section{Introduction}

The Brownian Loop Soup (BLS) [1] is an ideal gas of planar loops whose partition function can be formally written as

$$
Z=1+\sum_{n=1}^{\infty} \frac{\lambda^{n}}{n !} \prod_{i=1}^{n} \int \mu^{\mathrm{loop}}\left(\gamma_{i}\right) d \gamma_{i}
$$

where one integrates over all possible planar loops $\gamma$ and loops are weighted according to the Brownian loop measure $\mu^{\text {loop }}$ studied in [2]. In the BLS, the weights of loops are chosen in such a way as to make the model conformally invariant. Requiring conformal invariance essentially determines the measure $\mu^{\text {loop }}$ uniquely, up to a multiplicative parameter. Because of scale invariance, the total weight of all loops contained in any region of space is infinite, so $\mu^{\text {loop }}$ is an infinite measure (this is the reason it is only determined up to a multiplicative constant). In this paper, we adopt the standard normalization from [1].

A realization of the BLS consists of a countable collection of loops. In a bounded domain, when $\lambda \leq 1 / 2$ the loops form separate clusters. The collection of outer boundaries 
of the clusters of touching loops is a Conformal Loop Ensemble $\left(\mathrm{CLE}_{\kappa}\right)[3,4]$ with parameter $8 / 3<\kappa \leq 4$ related to the BLS intensity $\lambda$ according go the equation $\lambda=\frac{(3 \kappa-8)(6-\kappa)}{4 \kappa}$. CLEs are conjectured, and in some cases proven, to describe the scaling limit of cluster boundaries in various critical models of statistical mechanics, such as the critical Potts models for $q \in(1,4]$. For example, the continuum scaling limit of the collection of all macroscopic boundaries of critical Ising spin clusters corresponds to $\mathrm{CLE}_{3}$ and to a Brownian loop soup with $\lambda=1 / 4[5]$.

The intensity $\lambda$ of the Brownian loop soup is related to the central charge $c$ of the corresponding statistical mechanical model according to the relation $c=2 \lambda$. A discussion of the central charge of the Brownian loop soup can be found in section 6 of [6]. Since $c$ is continuous and can be less than one, this demonstrates that the model cannot be unitary for all values of $\lambda$ (the minimal models are the only unitary conformal field theories with $c<1$, and they come in a discrete series). Nevertheless, we were able to identify a set of conformal primary operators with positive conformal dimensions. These operators are exponentials of the form $e^{i \beta N(z)}$, where $\beta$ is a real number (the "charge") and $N(z)$ is an integer-valued operator that counts some characteristics of the loops.

In this paper, we will consider the case where $N(z)$ counts the "layering number." For each configuration of loops in the ensemble (1.1) defining the BLS, every loop is assigned a value \pm 1 with equal probability. Each loop that encircles the point $z$ contributes this value \pm 1 to $N(z) .{ }^{1}$ For instance, if $z$ lies inside two loops, $N(z)$ will take the value $\pm 2, \pm 1$, or 0 depending on what value was assigned to each loop (see figure 1 for an example). Note that these layering vertex operators are sensitive only to the outer boundary of each Brownian loop (figure 1b).

Because the BLS is a Poissonian ensemble of loops (that is, each loop is independent of the others), the probabilities of events in the BLS can be expressed in terms of $\mu^{\text {loop }}$. Specifically, we can obtain certain correlation functions in the BLS with suitable cutoffs simply by taking the exponential of $\lambda$ times the $\mu^{\text {loop }}$-weights of certain sets of loops.

In [6], we demonstrated that, after removing the cutoff, $e^{i \beta N(z)}$ is a scalar primary with conformal weights that are periodic functions of $\beta, \Delta=\bar{\Delta}=\frac{\lambda}{10}(1-\cos \beta)$, and that correlation functions of products of these operators vanish unless $\sum_{i} \beta_{i}=0 \bmod 2 \pi$. We computed the two- and three-point functions, but only up to multiplicative constants.

In this paper, we use the results of [8] to determine various expressions for the twoand three-point correlation functions. Together with a result of [9], we use these results to compute analytically and in closed form the full four-point correlation function of the layering vertex operators in the plane, as a function of the positions of the four points, the intensity $\lambda$, and the four charges $\beta_{i}$.

The results of [9] were obtained by taking the limit $n \rightarrow 0$ of the critical $O(n)$ model, which is conformally invariant and describes a single self-avoiding loop in this limit. As just mentioned, the layering vertex operators in the BLS are sensitive only to the outer boundary of each Brownian loop, and the outer boundary is by definition self-avoiding. Furthermore,

\footnotetext{
${ }^{1}$ In [7], two of us consider a generalization of this procedure where the loops are assigned more general random values.
} 


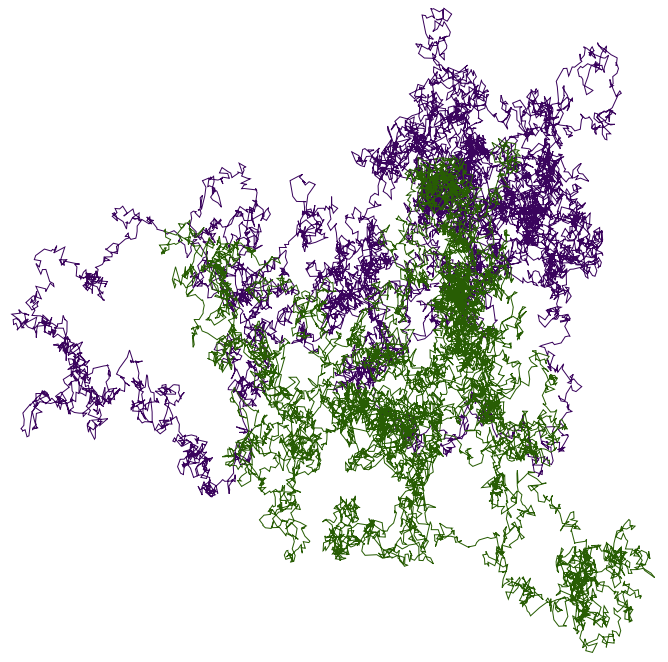

(a) Two Brownian loops.

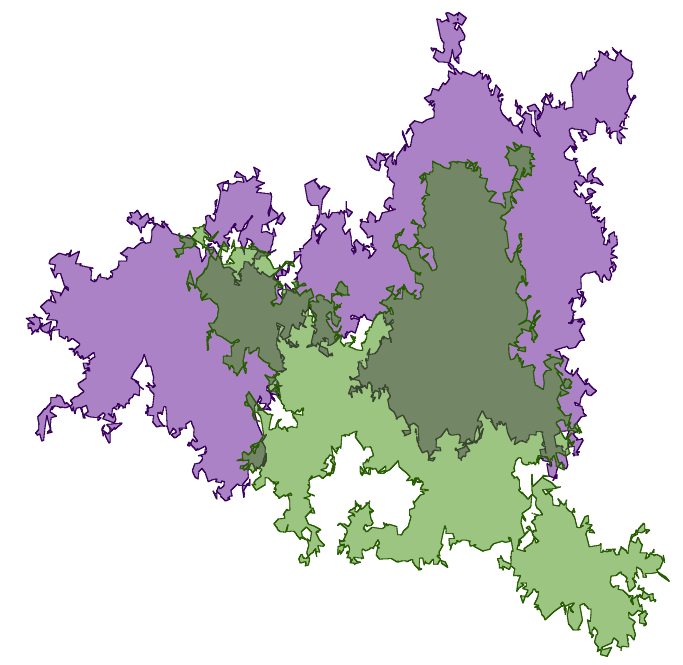

(b) The outer boundaries of the same loops.

Figure 1. Two random Brownian loops with identical parameters. For $z$ within its outer boundary, each loop contributes \pm 1 to the layering number $N(z)$, where the sign is chosen randomly and uniformly. If the purple loop is assigned +1 and the green loop $-1, N(z)=0$ for $z$ in the white or dark green areas, +1 in the purple, and -1 in the light green.

a result of [2] guarantees that there is a unique (up to an overall multiplicative constant) conformally invariant measure on self-avoiding loops (this is in fact the measure induced by $\mu^{\text {loop }}$ on the outer boundaries of Brownian loops). Therefore, since every loop in the BLS is independent of every other loop, we can use results from the $n \rightarrow 0$ of the critical $O(n)$ model to determine correlation functions in the BLS. Specifically, correlation functions of the operator $e^{i \pi N(z)}$ in the BLS are the exponentials of the intensity $\lambda$ times the correlation functions of the twist operators considered in [9], and correlation functions of more general BLS operators can be deduced as well.

With the four-point function in hand, we can expand it in conformal blocks. This reveals a new set of previously unknown conformal primary operators and their threepoint function coefficients with the layering vertex operators. The physical interpretation and meaning of these operators is left to future work.

Using the results of [8] we also compute the two-point function in the upper half-plane (subject to a certain boundary condition on the real axis), as a function of the positions of the two points, the intensity $\lambda$, and the two charges $\beta_{i}$. The results of [8] are rigorous and based on SLE theory [10]. They do not rely on the $n \rightarrow 0$ limit of the $O(n)$ model used in [9]. Limits of this two-point function help determine various constants in the four-point function. In particular, we obtain the interesting result that the three-point function coefficient for the canonically normalized layering vertex operators is exactly 1 . This is consistent with our results for the conformal block expansion of the four-point function. Here, by canonical normalization we mean that the operators are normalized so that the coefficients of the onepoint function in the upper half-plane and the two-point function in the plane are equal to 1.

We also determine the weights for Brownian loops to wind around one point and not another in the upper half-plane and full plane, and for several other configurations. 


\section{Summary and results}

Our main result is the derivation of new correlation functions of exponentials of the layering operators in the BLS. In [6], three of us showed that the conformal dimensions of the operators $e^{i \beta N(z)}$ are

$$
\Delta=\bar{\Delta}=\frac{\lambda}{10}(1-\cos \beta) .
$$

In this work, we obtain the two-point function of these operators in the upper half-plane with the boundary condition that any loop intersecting the real axis is erased, and the four-point function in the full plane.

The two-point function in the upper half-plane $\mathbb{H}$ (section 3 ) is given by

$$
\begin{aligned}
\left\langle\tilde{\mathcal{O}}_{\beta_{1}}\left(z_{1}\right) \tilde{\mathcal{O}}_{\beta_{2}}\left(z_{2}\right)\right\rangle_{\mathbb{H}}= & \left|z_{1}-z_{2}\right|^{-2\left(\Delta_{1}+\Delta_{2}-\Delta_{12}\right)}\left|z_{1}-\bar{z}_{2}\right|^{2\left(\Delta_{1}+\Delta_{2}-\Delta_{12}\right)}\left|z_{1}-\bar{z}_{1}\right|^{-2 \Delta_{1}}\left|z_{2}-\bar{z}_{2}\right|^{-2 \Delta_{2}} \\
& \times \exp \left[-\left(\Delta_{1}+\Delta_{2}-\Delta_{12}\right)(1-\sigma)_{3} F_{2}\left(1,1, \frac{4}{3} ; 2, \frac{5}{3} ; 1-\sigma\right)\right],
\end{aligned}
$$

where $\tilde{\mathcal{O}}_{\beta}(z) \propto e^{i \beta N(z)}$ are exponentials of layering operators normalized so that $\left\langle\tilde{\mathcal{O}}_{\beta}(z)\right\rangle_{\mathbb{H}}=|z-\bar{z}|^{-2 \Delta}$, and $\sigma, \Delta_{i}$, and $\Delta_{i j}$ are defined in (3.6) and (3.11)

The four-point function of these operators in the full plane $\mathbb{C}$ (section 5 ) is given by

$$
\begin{aligned}
\left\langle\prod_{i=1}^{4} \mathcal{O}_{\beta_{i}}\left(z_{i}\right)\right\rangle_{\mathbb{C}}= & \exp \left[-2 A(x)\left(\sum_{i=1}^{4} \Delta_{i}-\sum_{j=2}^{4} \Delta_{1 j}\right)\right]\left|\frac{z_{13} z_{24}}{z_{12} z_{34}}\right|^{-2 \Delta_{12}}\left|\frac{z_{13} z_{24}}{z_{14} z_{23}}\right|^{-2 \Delta_{14}} \\
& \times\left|\frac{z_{12} z_{14}}{z_{24}}\right|^{-2 \Delta_{1}}\left|\frac{z_{12} z_{23}}{z_{13}}\right|^{-2 \Delta_{2}}\left|\frac{z_{23} z_{34}}{z_{24}}\right|^{-2 \Delta_{3}}\left|\frac{z_{14} z_{34}}{z_{13}}\right|^{-2 \Delta_{4}},
\end{aligned}
$$

with $z_{i j}=\left|z_{i}-z_{j}\right|$ and

$$
\begin{aligned}
A(x)= & \frac{1}{4}\left[x_{3} F_{2}\left(1,1, \frac{4}{3} ; 2, \frac{5}{3} ; x\right)+\bar{x}_{3} F_{2}\left(1,1, \frac{4}{3} ; 2, \frac{5}{3} ; \bar{x}\right)\right] \\
& -\frac{2 \cdot 2^{\frac{1}{3}} \pi^{2}}{\sqrt{3} \Gamma\left(\frac{1}{6}\right)^{2} \Gamma\left(\frac{4}{3}\right)^{2}}|x(1-x)|^{\frac{2}{3}}\left|{ }_{2} F_{1}\left(\frac{2}{3}, 1: \frac{4}{3} ; x\right)\right|^{2},
\end{aligned}
$$

where the operators are canonically normalized, so that $\left\langle\mathcal{O}_{\beta_{1}}\left(z_{1}\right) \mathcal{O}_{\beta_{2}}\left(z_{2}\right)\right\rangle_{\mathbb{C}}=\left|z_{1}-z_{2}\right|^{-2 \Delta_{1}}$, and $x$ is the cross-ratio (5.5).

All $n$-point functions in the full plane vanish unless a (periodic) charge conservation condition is satisfied:

$$
\sum_{i=1}^{n} \beta_{i}=2 \pi k, \quad k \in \mathbb{Z}
$$

The conformal block expansion of the four-point function (2.4) (section 6) reveals the spectrum of conformal primaries and associated three-point function coefficients. We find an apparently infinite new set of primary operators of integer spin in the BLS, with conformal dimensions

$$
\begin{aligned}
& \Delta^{\left(p, p^{\prime}\right)}=\frac{\lambda}{10}\left(1-\cos \left(\beta_{1}+\beta_{2}\right)\right)+\frac{p}{3} \\
& \bar{\Delta}^{\left(p, p^{\prime}\right)}=\frac{\lambda}{10}\left(1-\cos \left(\beta_{1}+\beta_{2}\right)\right)+\frac{p^{\prime}}{3},
\end{aligned}
$$

where $p$ and $p^{\prime}$ are non-negative integers satisfying $p-p^{\prime}=0 \bmod 3$. 
Weights for Brownian loops to encircle various subsets of points in the plane or upper half-plane can be found in (B.1), (B.3), and (5.8).

\subsection{Motivation and previous work}

In [11], Freivogel and Kleban considered a toy model intended to capture the late-time physics of cosmic bubble nucleation in eternally inflating or de Sitter spacetime. In a spacetime with one time and two space dimensions, these bubbles will be disks (with random fluctuations to their shape) that expand exponentially after their nucleation due to the expansion of the ambient spacetime. This turns out to imply that on a late time slice the distribution of disks will be invariant under translations, scale transformations, rotations, and special conformal transformations [12]. This "disk soup" has intensity $\lambda_{\mathrm{FK}}$ that is equal to the bubble production rate per Hubble time per Hubble volume.

It is widely believed that theories with Poincaré and scale invariance are fully conformally invariant. However, the disk soup model of [11] appears to be an exception. Operators of the form $e^{i \beta N(z)}$, where $N(z)$ now counts the number of disks that overlap the point $z$ (the "layering" operator in the parlance of this paper) exhibit the behavior of primary operators with dimension $\Delta=\bar{\Delta}=\frac{\pi}{2} \lambda_{\mathrm{FK}}(1-\cos \beta)-$ explicit computation shows that their two- and three-point functions have the appropriate $z$-dependence. However, the four-point function, while crossing symmetric, is a non-analytic function of the $z_{i}$. For this reason there is no conformal block expansion. Presumably, this is because general conformal transformations do not map disks into disks, so the disk distribution is not invariant under local conformal transformations.

A primary motivation for [6] was to obtain a full-fledged conformal field theory by replacing the disk distribution of [11] with the Brownian loop measure [2]. The analog of the disk model with the disk distribution replaced by the Brownian loop measure is precisely the BLS [1]. Since the BLS is known to be locally conformally invariant, a theory defined by it should be a full-fledged local conformal field theory. While [6] demonstrated that the exponentials of the (loop) layering operators are conformal primary operators, we were unable to compute the three-point function coefficients or four-point correlation functions.

In this work, we take a major step beyond [6] by obtaining explicit results for the four-point function in the plane - which indeed is an analytic function of the cross ratio and has a conformal block expansion - as well as the three-point function coefficients, and the two-point function on the upper half-plane.

Models with similar properties of our model have been studied in the literature. An example of an infinite discrete set of primary operators is found in Liouville theory, which also allows a description in terms of loop ensembles [13]. A very powerful tool in the study of Liouville theory is the DOZZ formula $[14,15]$ and its extensions which allow for analytic expressions of the three-point function coefficients.

As mentioned previously, other conformal theories in two dimensions that can be understood in terms of random loops are the so-called random cluster models, of which the $q$-state Potts model and percolation are special cases. Some recent results in these theories include $[16,17]$. 
At this point it is not evident how the BLS model studied here relates to other known $2 \mathrm{D}$ conformal theories, except in its explicit connection to the ensemble of a single selfavoiding random loop that can be obtained from the $n \rightarrow 0$ limit of the $O(n)$ model. $^{2}$ However the periodic-in- $\beta$ conformal dimensions (2.1) and the infinite set of primary operators with integer spin but dimensions separated by integers/3 (2.6) that we identify via the conformal block expansion appear to be novel features with no known analogs. It would be very interesting to explore the connection to the $O(n)$ model in greater depth, and we intend to do so in future work.

\section{The two-point function in the upper half-plane}

In this section, we will use the results of $[6,8]$ to derive the general two-point function of layering vertex operators in the upper half-plane $\mathbb{H}$. In this section, and in the rest of the paper, we will make extensive use of a main result from [6]:

$$
\left\langle\prod_{j=1}^{n} e^{i \beta_{j} N\left(z_{j}\right)}\right\rangle=\prod_{S \subseteq\left\{z_{1}, \ldots, z_{n}\right\}} \exp \left[-\lambda \alpha\left(S \mid S^{c}\right)\left(1-\cos \sum_{k \in I_{S}} \beta_{k}\right)\right] .
$$

Here the product is over all nonempty subsets $S \subset\left\{z_{1}, \ldots, z_{n}\right\}$ and $I_{S}$ denotes the set of indices corresponding to the points of $\left\{z_{1}, \ldots, z_{n}\right\}$ contained in $S$. The $\alpha\left(S \mid S^{c}\right)$ are the weights, according to the Brownian loop measure, of the sets of loops that encircle the points in $S$ but not those in $S^{c}$. The loops need to be contained in some domain $D$, which in this paper is either the upper half-plane or the full plane. We will denote weights in the upper half-plane by $\alpha_{\mathbb{H}}$ and weights in the full plane simply by $\alpha$, and correlation functions by $\langle\ldots\rangle_{\mathbb{H}}$ and $\langle\ldots\rangle_{\mathbb{C}}$, respectively. In words, (3.1) states that a general $n$-point function of layering vertex operators in the BLS equals the exponential of terms consisting of the weights for loops that encircle various subsets of the points times the associated conformal weights.

The two-point function in any simply connected domain of the plane can be obtained from that in $\mathbb{H}$ by a conformal transformation, so computing the two-point function in $\mathbb{H}$ in principle gives the two-point function in a domain of any shape. The boundary condition is that all loops must be confined entirely to the interior of $\mathbb{H}$ (that is, one could consider the BLS on the full plane and remove all loops that intersect the lower half-plane).

Our strategy is to first find the weights of loops that encircle one or both points in $\mathbb{H}$. Once we have these weights we can immediately write down the two-point function for general $\beta_{i}$ using (3.1). We adopt a notation related to that of [6]. For two points $z_{1}=x_{1}+i y_{1}, z_{2}=x_{2}+i y_{2} \in \mathbb{C}$ let

$$
\begin{aligned}
\alpha_{\mathbb{H}}\left(z_{1} \mid z_{2}\right) & =\mu^{\operatorname{loop}}\left\{\gamma: \operatorname{diam}(\gamma)>\delta, \gamma \subseteq \mathbb{H}, z_{1} \in \bar{\gamma}, z_{2} \notin \bar{\gamma}\right\} \\
\alpha_{\mathbb{H}}\left(z_{1}, z_{2}\right) & =\mu^{\operatorname{loop}}\left\{\gamma: \operatorname{diam}(\gamma)>\delta, \gamma \subseteq \mathbb{H}, z_{1}, z_{2} \in \bar{\gamma}\right\} .
\end{aligned}
$$

\footnotetext{
${ }^{2}$ As mentioned above, the precise relation is that correlation functions of the operator $e^{i \pi N(z)}$ in the BLS are the exponential of the intensity $\lambda$ times the correlation functions of the twist operators considered in [9] that were obtained from the $n \rightarrow 0$ limit of the $O(n)$ model.
} 
Here $\delta>0$ is a short-distance regulator that we will later take to zero, $\gamma$ is a loop (left panel of figure 1), $\bar{\gamma}$ is the interior of $\gamma$ (right panel of figure 1 , shaded region), and $\operatorname{diam}(\gamma)$ is its diameter (the largest distance between any two points on the loop).

In general, the weights of loops that encircle only one point (such as (3.2), first line) diverge as $\delta \rightarrow 0$ due to contributions from arbitrarily small loops, infinitely many of which encircle any given point. Weights of loops that encircle two or more points (such as (3.2), second line) are finite as $\delta \rightarrow 0$ because only loops whose diameter is larger or equal to the distance between the two closest points encircle them.

For $\left|z_{1}-z_{2}\right| \geq \delta$, using equations (1.5) and (1.3) of [8] (the first multiplied by $6 \pi / 5$ ), we have that

$$
\begin{aligned}
\alpha_{\mathbb{H}}\left(z_{1}, z_{2}\right)= & -\frac{\pi}{5 \sqrt{3}}-\frac{1}{10} \eta_{3} F_{2}\left(1,1, \frac{4}{3} ; 2, \frac{5}{3} ; \eta\right)-\frac{1}{10} \log (\eta(\eta-1)) \\
& +\frac{\Gamma\left(\frac{2}{3}\right)^{2}}{5 \Gamma\left(\frac{4}{3}\right)}(\eta(\eta-1))^{\frac{1}{3}}{ }_{2} F_{1}\left(1, \frac{2}{3} ; \frac{4}{3}, \eta\right) \\
= & -\frac{1}{10}\left[\log \sigma+(1-\sigma)_{3} F_{2}\left(1,1, \frac{4}{3} ; 2, \frac{5}{3} ; 1-\sigma\right)\right]
\end{aligned}
$$

where

$$
\eta=-\frac{\left(x_{1}-x_{2}\right)^{2}+\left(y_{1}-y_{2}\right)^{2}}{4 y_{1} y_{2}}=\frac{\left(z_{1}-z_{2}\right)\left(\bar{z}_{1}-\bar{z}_{2}\right)}{\left(z_{1}-\bar{z}_{1}\right)\left(z_{2}-\bar{z}_{2}\right)}
$$

and

$$
\sigma=\frac{\left|z_{1}-z_{2}\right|^{2}}{\left|z_{1}-\bar{z}_{2}\right|^{2}}=\frac{\left(x_{1}-x_{2}\right)^{2}+\left(y_{1}-y_{2}\right)^{2}}{\left(x_{1}-x_{2}\right)^{2}+\left(y_{1}+y_{2}\right)^{2}} .
$$

Notice, for future reference, that $\eta-1=\frac{\left(z_{1}-\bar{z}_{2}\right)\left(\bar{z}_{1}-z_{2}\right)}{\left(z_{1}-\bar{z}_{1}\right)\left(z_{2}-\bar{z}_{2}\right)}$.

We can use (3.4) and properties of the weight of the loops around $z_{1}$ in the Brownian loop measure to get an expression for $\alpha_{\mathbb{H}}\left(z_{1} \mid z_{2}\right)$. By using scale and translation invariance of the Brownian loop measure, and Lemma A1 of [6], if $\delta \leq y_{1}$, we have

$$
\begin{aligned}
\alpha_{\mathbb{H}}\left(z_{1}\right) & =\mu^{\operatorname{loop}}\left\{\gamma: \operatorname{diam}(\gamma)>\delta, \gamma \subseteq \mathbb{H}, z_{1} \in \bar{\gamma}\right\} \\
& =\frac{1}{5} \log \frac{y_{1}}{\delta}+\hat{\alpha}=\frac{1}{5} \log \frac{\left|z_{1}-\bar{z}_{1}\right|}{2 \delta}+\hat{\alpha},
\end{aligned}
$$

where $\hat{\alpha}=\mu^{\operatorname{loop}}\left\{\gamma: \operatorname{diam}(\gamma)>1, \gamma \subseteq \mathbb{H}, z_{1} \in \bar{\gamma}\right\}$ is the weight of the loops with diameter greater than or equal to 1 contained in $\mathbb{H}$ and winding around the point $z=i$. On the other hand, if $\left|z_{1}-z_{2}\right| \geq \delta$, then

$$
\alpha_{\mathbb{H}}\left(z_{1}\right)=\alpha_{\mathbb{H}}\left(z_{1} \mid z_{2}\right)+\alpha_{\mathbb{H}}\left(z_{1}, z_{2}\right) ;
$$

hence, for $\delta \leq \min \left(\left|z_{1}-z_{2}\right|,\left|z_{1}-\bar{z}_{1}\right| / 2\right)$ we finally obtain

$$
\alpha_{\mathbb{H}}\left(z_{1} \mid z_{2}\right)=-\alpha_{\mathbb{H}}\left(z_{1}, z_{2}\right)+\frac{1}{5} \log \left|z_{1}-\bar{z}_{1}\right|-\frac{1}{5} \log (2 \delta)+\hat{\alpha} .
$$

Using (3.1) and denoting the conformal dimensions by

$$
\begin{aligned}
\Delta_{j} & =\frac{\lambda}{10}\left(1-\cos \beta_{j}\right) \\
\text { and } \quad \Delta_{i j} & =\frac{\lambda}{10}\left(1-\cos \left(\beta_{i}+\beta_{j}\right)\right),
\end{aligned}
$$


we have

$$
\begin{aligned}
\left\langle e^{i \beta_{1} N\left(z_{1}\right)} e^{i \beta_{2} N\left(z_{2}\right)}\right\rangle_{\mathbb{H}} \\
=\exp \left[-\lambda\left(\left(1-\cos \beta_{1}\right) \alpha_{\mathbb{H}}\left(z_{1} \mid z_{2}\right)+\left(1-\cos \beta_{2}\right) \alpha_{\mathbb{H}}\left(z_{2} \mid z_{1}\right)+\left(1-\cos \left(\beta_{1}+\beta_{2}\right)\right) \alpha_{\mathbb{H}}\left(z_{1}, z_{2}\right)\right)\right] \\
=\left(2 \delta e^{-5 \hat{\alpha}}\right)^{2\left(\Delta_{1}+\Delta_{2}\right)}\left|z_{1}-z_{2}\right|^{-2\left(\Delta_{1}+\Delta_{2}-\Delta_{12}\right)}\left|z_{1}-\bar{z}_{2}\right|^{2\left(\Delta_{1}+\Delta_{2}-\Delta_{12}\right)}\left|z_{1}-\bar{z}_{1}\right|^{-2 \Delta_{1}}\left|z_{2}-\bar{z}_{2}\right|^{-2 \Delta_{2}} \\
\quad \times \exp \left[-\left(\Delta_{1}+\Delta_{2}-\Delta_{12}\right)(1-\sigma)_{3} F_{2}\left(1,1, \frac{4}{3} ; 2, \frac{5}{3} ; 1-\sigma\right)\right] .
\end{aligned}
$$

This equation implies that the canonical normalization in the upper half-plane is obtained by multiplying the normalizing factor $\delta^{2 \Delta_{j}}$ used in [6] by $\left(2 e^{-5 \hat{\alpha}}\right)^{2 \Delta_{j}}$. Defining $\tilde{\mathcal{O}}_{\beta}(z) \equiv$ $\left(2 \delta e^{-5 \hat{\alpha}}\right)^{-2 \Delta} e^{i \beta N(z)}$, the two-point function becomes

$$
\begin{aligned}
\left\langle\tilde{\mathcal{O}}_{\beta_{1}}\left(z_{1}\right) \tilde{\mathcal{O}}_{\beta_{2}}\left(z_{2}\right)\right\rangle_{\mathbb{H}} \equiv & \lim _{\delta \rightarrow 0} \frac{\left\langle e^{i \beta_{1} N\left(z_{1}\right)} e^{i \beta_{2} N\left(z_{2}\right)}\right\rangle_{\mathbb{H}}}{\left(2 \delta e^{-5 \hat{\alpha}}\right)^{2\left(\Delta_{1}+\Delta_{2}\right)}} \\
= & \left|z_{1}-z_{2}\right|^{-2\left(\Delta_{1}+\Delta_{2}-\Delta_{12}\right)}\left|z_{1}-\bar{z}_{2}\right|^{2\left(\Delta_{1}+\Delta_{2}-\Delta_{12}\right)}\left|z_{1}-\bar{z}_{1}\right|^{-2 \Delta_{1}}\left|z_{2}-\bar{z}_{2}\right|^{-2 \Delta_{2}} \\
& \times \exp \left[-\left(\Delta_{1}+\Delta_{2}-\Delta_{12}\right)(1-\sigma)_{3} F_{2}\left(1,1, \frac{4}{3} ; 2, \frac{5}{3} ; 1-\sigma\right)\right] .
\end{aligned}
$$

Note that the one-point function can be immediately obtained from (3.13) by setting, e.g., $\beta_{2}=0$ :

$$
\left\langle\tilde{\mathcal{O}}_{\beta_{1}}\left(z_{1}\right)\right\rangle_{\mathbb{H}}=\left|z_{1}-\bar{z}_{1}\right|^{-2 \Delta_{1}}
$$

\section{The two- and three-point functions in the full plane}

In this section, we compute the two- and three-point functions of layering vertex operators in the full plane. A major difference from the half-plane is that in the full plane all correlation functions go to zero because of the contribution from large loops, unless the charge conservation condition

$$
\sum_{i=1}^{n} \beta_{i}=2 \pi k, \quad k \in \mathbb{Z}
$$

is satisfied $[6,11]$. This is reminiscent of momentum or charge conservation for the vertex operators of the free boson, where the condition arises from integration over the zero mode.

Note that this condition would also be clearly necessary were we to define these correlation functions on the sphere rather than the plane, because on a sphere a loop that covers a subset of points can equally well be interpreted as a loop that covers the complement of that set (on a compact space there is no notion of the "inside" versus the "outside" of the loop). Since the plane and the sphere are conformally equivalent, (4.1) is necessary for consistency (cf. section 5).

The $z_{i}$ dependence of the two- and three-point functions in the full plane follow from conformal invariance and the fact that the layering vertex operators are conformal primaries [6]. However, this argument does not fix the constant prefactors, which were not computed in [6]. As we show in appendix A, by taking the limit that the points are far from 
the boundary, we can use our results from the upper half-plane to determine the multiplicative prefactors left undetermined in [6]. We find that the most convenient normalization in the plane is

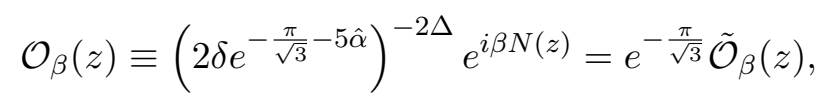

where again $\hat{\alpha}$ is a constant equal to the weight of loops in the upper half-plane with diameter larger than 1 and that encircle $z=i$. Comparing (4.2) to (3.13) shows that the difference between the canonical normalizations in the half-plane and in the plane is simply the factor $e^{\frac{2 \pi}{\sqrt{3}} \Delta}$.

The two- and three-point functions of $\mathcal{O}_{\beta}(z)$ in the limit $\delta \rightarrow 0$ are

$$
\left\langle\mathcal{O}_{\beta_{1}}\left(z_{1}\right) \mathcal{O}_{\beta_{2}}\left(z_{2}\right)\right\rangle_{\mathbb{C}}=\left|z_{1}-z_{2}\right|^{-4 \Delta_{1}}
$$

and

$$
\begin{aligned}
& \left\langle\mathcal{O}_{\beta_{1}}\left(z_{1}\right) \mathcal{O}_{\beta_{2}}\left(z_{2}\right) O_{\beta_{3}}\left(z_{3}\right)\right\rangle_{\mathbb{C}} \\
& \quad=\left|z_{1}-z_{2}\right|^{-2\left(\Delta_{1}+\Delta_{2}-\Delta_{3}\right)}\left|z_{1}-z_{3}\right|^{-2\left(\Delta_{1}+\Delta_{3}-\Delta_{2}\right)}\left|z_{2}-z_{3}\right|^{-2\left(\Delta_{2}+\Delta_{3}-\Delta_{1}\right)}
\end{aligned}
$$

as shown in appendix A. The calculation leading to (4.4) is possible because, when the three-point function is expressed in terms of $\alpha\left(z_{1}, z_{2}\right)$ and $\alpha\left(z_{1}, z_{2}, z_{3}\right)$, the coefficient of $\alpha\left(z_{1}, z_{2}, z_{3}\right)$ turns out to be zero thanks to charge conservation (see appendix A). Remarkably, the three-point function coefficients are precisely 1 for all values of the $\beta_{i}$ satisfying (4.1).

In the notation of [6], these results are equivalent to

$$
C_{2}=\left(2 e^{-\frac{\pi}{\sqrt{3}}-5 \hat{\alpha}}\right)^{2\left(\Delta_{1}+\Delta_{2}\right)}, \quad C_{3}=\left(2 e^{-\frac{\pi}{\sqrt{3}}-5 \hat{\alpha}}\right)^{2\left(\Delta_{1}+\Delta_{2}+\Delta_{3}\right)} .
$$

\section{The general four-point function in the plane}

In this section, we will compute the general four-point function for the layering operators in the whole plane. As before, the computation uses (3.1) and the derivation of the weights of various collections of loops. But while all results presented so far were mathematically rigorous, this time we rely on a non-rigorous result of [9] obtained taking the $n \rightarrow 0$ limit of quantities computed for the $O(n)$ model. We also point out, however, that in appendix $\mathrm{B}$ we give a rigorous derivation of a similar result from [9], obtained there with the same $n \rightarrow 0$ technique. This provides additional evidence in favor of the validity of the $O(n)$ expansion and $n \rightarrow 0$ limit used in [9]. 
Consider four points $z_{1}, z_{2}, z_{4}, z_{4}$ and assume in what follows that the letters $i, j, k, \ell \in$ $\{1,2,3,4\}$ are always different. Using (3.1), the four-point function can be written as

$$
\begin{aligned}
&\left\langle\prod_{i=1}^{4} e^{i \beta_{i} N\left(z_{i}\right)}\right\rangle_{\mathbb{C}}=\exp \left[-\lambda\left(\sum_{i=1}^{4}\left(1-\cos \beta_{i}\right) \alpha\left(z_{i} \mid z_{j}, z_{k}, z_{\ell}\right)\right.\right. \\
&+\sum_{\substack{i, j=1 \\
i<j}}^{4}\left(1-\cos \left(\beta_{i}+\beta_{j}\right)\right) \alpha\left(z_{i}, z_{j} \mid z_{k}, z_{\ell}\right) \\
&+\sum_{i=1}^{4}\left(1-\cos \left(\beta_{j}+\beta_{k}+\beta_{\ell}\right)\right) \alpha\left(z_{j}, z_{k}, z_{\ell} \mid z_{i}\right) \\
&\left.\left.+\left(1-\cos \left(\beta_{1}+\beta_{2}+\beta_{3}+\beta_{4}\right)\right) \alpha\left(z_{1}, z_{2}, z_{3}, z_{4}\right)\right)\right],
\end{aligned}
$$

where the weights $\alpha$ of loops encircling points in the full plane are defined analogously to (3.2). Imposing the charge conservation condition (4.1), the four-point function becomes

$$
\begin{aligned}
\left\langle\prod_{i=1}^{4} e^{i \beta_{i} N\left(z_{i}\right)}\right\rangle_{\mathbb{C}}=\exp [-\lambda( & \sum_{i=1}^{4}\left(1-\cos \beta_{i}\right) \alpha_{\mathbb{S}}\left(z_{i} \mid z_{j}, z_{k}, z_{\ell}\right) \\
& \left.\left.+\sum_{j=2}^{4}\left(1-\cos \left(\beta_{1}+\beta_{j}\right)\right) \alpha_{\mathbb{S}}\left(z_{1}, z_{j} \mid z_{k}, z_{\ell}\right)\right)\right],
\end{aligned}
$$

where we introduced the weights

$$
\alpha_{\mathbb{S}}\left(S \mid S^{c}\right) \equiv \alpha\left(S \mid S^{c}\right)+\alpha\left(S^{c} \mid S\right)
$$

for subsets of points $S \subseteq\left\{z_{1}, z_{2}, z_{3}, z_{4}\right\}$, with $S^{c}$ denoting the complement of $S$. For instance, $\alpha_{\mathbb{S}}\left(z_{1} \mid z_{2}, z_{3}, z_{4}\right)=\alpha\left(z_{1} \mid z_{2}, z_{3}, z_{4}\right)+\alpha\left(z_{2}, z_{3}, z_{4} \mid z_{1}\right)$.

As previously mentioned, if we consider the BLS on a sphere rather than the plane, charge conservation is necessary for consistency because there is no distinction between the inside and outside of a loop on a sphere. Another implication of this fact is that both "sides" of the loop must contribute equally to the correlation functions. Under stereographic projection to the plane, the "outside" of the loop is the side that contains the point that projects to infinity of the plane, but it remains the case that both the inside and the outside must contribute. This explains why only the paired weights $\alpha_{\mathbb{S}}$ appear in (5.2).

There are a total of seven pairs $\alpha_{\mathbb{S}}$ that contribute. Six of these can be determined from the results we have already obtained for the two-point functions (we can also obtain relations from the three-point functions, but they are not independent). For the seventh relation we will use a result of [9].

Choosing $\beta_{1}=\beta_{2}=\pi, \beta_{3}=\beta_{4}=0$ in (5.2) reproduces the two-point function. Hence comparing (5.2) with (3.1) gives the relation

$$
\begin{array}{r}
\alpha_{\mathbb{S}}\left(z_{1} \mid z_{2}, z_{3}, z_{4}\right)+\alpha_{\mathbb{S}}\left(z_{2} \mid z_{1}, z_{3}, z_{4}\right)+\alpha_{\mathbb{S}}\left(z_{1}, z_{3} \mid z_{2}, z_{4}\right)+\alpha_{\mathbb{S}}\left(z_{1}, z_{4} \mid z_{2}, z_{3}\right) \\
=2 \alpha\left(z_{1} \mid z_{2}\right)=\frac{2}{5} \log \left|z_{1}-z_{2}\right|+2 Q,
\end{array}
$$


where we used (B.1) in the last equality and $Q$ is defined in (B.2). Five other independent equations can be obtained by choosing other pairs of the $\beta_{i}$ equal to $\pi$ and 0 .

The system of six equations we obtain from (5.4) and its permutations has rank six. An additional independent relation is necessary to solve for the seven $\alpha_{\mathbb{S}}$, and is provided by [9]. Defining $z_{j k}=z_{j}-z_{k}$, the cross-ratio

$$
x=\frac{z_{12} z_{34}}{z_{13} z_{24}}, \quad 1-x=\frac{z_{14} z_{23}}{z_{13} z_{24}},
$$

and the function

$$
\begin{aligned}
A(x)= & \frac{1}{4}\left[x_{3} F_{2}\left(1,1, \frac{4}{3} ; 2, \frac{5}{3} ; x\right)+\bar{x}_{3} F_{2}\left(1,1, \frac{4}{3} ; 2, \frac{5}{3} ; \bar{x}\right)\right] \\
& -\left.\left.\frac{2 \cdot 2^{\frac{1}{3}} \pi^{2}}{\sqrt{3} \Gamma\left(\frac{1}{6}\right)^{2} \Gamma\left(\frac{4}{3}\right)^{2}}|x(1-x)|^{\frac{2}{3}}\right|_{2} F_{1}\left(\frac{2}{3}, 1 ; \frac{4}{3} ; x\right)\right|^{2},
\end{aligned}
$$

equations (21) and (22) of [9] imply that

$$
\sum_{i=1}^{4} \alpha_{\mathbb{S}}\left(z_{i} \mid z_{j}, z_{k}, z_{\ell}\right)=P\left(\log \left|x z_{23} z_{14}\right|+2 A(x)\right)+4(Q+R) .
$$

Cardy and Gamsa derived this result by solving a linear differential equation that does not fix the overall normalization or the additive constant, so we have included an overall coefficient $P$, and retained an additive constant that we denote $4(Q+R)$ for future convenience (we will see shortly that $R=0$, and $Q$ is defined by (B.2)).

To determine $P$ we can examine the scaling behavior of the four-point function (5.2) where we set $\beta_{i}=\pi$ and therefore $\Delta_{i}=\lambda / 5, \Delta_{i j}=0$. In general, if $O_{\Delta, \Delta}$ is a primary of dimension $(\Delta, \Delta)$, rescaling $z_{i} \rightarrow \rho z_{i}$ takes $\log \left\langle O_{\Delta, \Delta}^{4}\right\rangle \rightarrow-8 \Delta \log \rho+\log \left\langle O_{\Delta, \Delta}^{4}\right\rangle$. Using (5.2), this fixes $P=2 / 5$.

We now have seven independent equations for the seven $\alpha_{\mathbb{S}}$. The solutions are

$$
\begin{aligned}
& \alpha_{\mathbb{S}}\left(z_{1} \mid z_{2}, z_{3}, z_{4}\right)=\frac{1}{5}\left(\log \left|\frac{z_{12} z_{14}}{z_{24}}\right|+A(x)\right)+Q+R \\
& \alpha_{\mathbb{S}}\left(z_{2} \mid z_{1}, z_{3}, z_{4}\right)=\frac{1}{5}\left(\log \left|\frac{z_{12} z_{23}}{z_{13}}\right|+A(x)\right)+Q+R \\
& \alpha_{\mathbb{S}}\left(z_{3} \mid z_{1}, z_{2}, z_{4}\right)=\frac{1}{5}\left(\log \left|\frac{z_{23} z_{34}}{z_{24}}\right|+A(x)\right)+Q+R \\
& \alpha_{\mathbb{S}}\left(z_{4} \mid z_{1}, z_{2}, z_{3}\right)=\frac{1}{5}\left(\log \left|\frac{z_{14} z_{34}}{z_{13}}\right|+A(x)\right)+Q+R \\
& \alpha_{\mathbb{S}}\left(z_{1}, z_{2} \mid z_{3}, z_{4}\right)=-\frac{1}{5}(\log |x|+A(x))-R \\
& \alpha_{\mathbb{S}}\left(z_{1}, z_{3} \mid z_{2}, z_{4}\right)=-\frac{1}{5} A(x)-R \\
& \alpha_{\mathbb{S}}\left(z_{1}, z_{4} \mid z_{2}, z_{3}\right)=-\frac{1}{5}(\log |1-x|+A(x))-R
\end{aligned}
$$

We can now show that $R=0$. Consider the four points $z_{i}$ arranged in a rectangle in cyclical order. If we let a pair of points approach the other pair by taking $z_{1} \rightarrow z_{4}$ and 
$z_{2} \rightarrow z_{3}$ it is clear that $\alpha\left(z_{1}, z_{3} \mid z_{2}, z_{4}\right) \rightarrow 0$, since the measure of loops passing between the pairs of points goes to zero. In the same limit we have that $x \rightarrow 1, A(x) \rightarrow 0$. Comparing this with (5.8f) shows that $R=0$. The weights $(5.8 \mathrm{e})-(5.8 \mathrm{~g})$ coincide with those given in (29)-(31) of [9], after multiplication by an overall factor $6 \pi / 5$.

This allows us to write the fully general, normalized four-point function as

$$
\begin{aligned}
\left\langle\prod_{i=1}^{4} \mathcal{O}_{\beta_{i}}\left(z_{i}\right)\right\rangle_{\mathbb{C}}= & \lim _{\delta \rightarrow 0}\left(2 \delta e^{-5 \bar{\alpha}-\frac{\pi}{\sqrt{3}}}\right)^{-2 \sum_{j=1}^{4} \Delta_{j}}\left\langle\prod_{i=1}^{4} e^{i \beta_{i} N\left(z_{i}\right)}\right\rangle_{\mathbb{C}} \\
= & \exp \left[-2 A(x)\left(\sum_{i=1}^{4} \Delta_{i}-\sum_{j=2}^{4} \Delta_{1 j}\right)\right]\left|\frac{z_{13} z_{24}}{z_{12} z_{34}}\right|^{-2 \Delta_{12}}\left|\frac{z_{13} z_{24}}{z_{14} z_{23}}\right|^{-2 \Delta_{14}} \\
& \times\left|\frac{z_{12} z_{14}}{z_{24}}\right|^{-2 \Delta_{1}}\left|\frac{z_{12} z_{23}}{z_{13}}\right|^{-2 \Delta_{2}}\left|\frac{z_{23} z_{34}}{z_{24}}\right|^{-2 \Delta_{3}}\left|\frac{z_{14} z_{34}}{z_{13}}\right|^{-2 \Delta_{4}}
\end{aligned}
$$

where $A(x)$ is defined by (5.6) and the $\Delta_{i}, \Delta_{i j}$ by (3.11).

With some algebra and using the identity

$$
A(x)-A(1 / x)+\log |x|=0
$$

one can check that the four-point function is invariant under exchange of any pair of indices, establishing crossing invariance.

\subsection{Free-field limit}

There is a limit in which the correlators in the full plane become those of free field vertex operators (the same limit was considered and the same result obtained in [11], for the disk model studied there). Consider taking $\beta_{i} \rightarrow 0$ and $\lambda \rightarrow \infty$ with the product $\lambda \beta_{i}^{2}$ fixed. We define the field $\psi$ by $\beta N(z)=\sqrt{2} \gamma \psi(z)$ with

$$
\gamma=\sqrt{\frac{\lambda}{20}} \beta
$$

such that the conformal dimension of the operator $e^{i \beta N(z)}=e^{i \sqrt{2} \gamma \psi(z)}$ becomes

$$
\Delta=\frac{\lambda}{10}(1-\cos \beta) \rightarrow \frac{\lambda}{20} \beta^{2}=\gamma^{2} .
$$

This is the correct dimension for a canonically normalized free-field vertex operator $e^{i \sqrt{2} \gamma \psi}$. Now consider (5.9) and note that

$$
\sum_{i=1}^{4} \Delta_{i}-\sum_{j=2}^{4} \Delta_{1 j} \rightarrow \sum_{i=1}^{4} \gamma_{i}^{2}-\sum_{j=2}^{4}\left(\gamma_{1}+\gamma_{j}\right)^{2}=0,
$$

where we used the fact that $\sum_{i=1}^{4} \gamma_{i}=0$. Therefore the factor in (5.9) involving hypergeometric functions goes to 1 in this limit, and the remainder reduces immediately to the correct form for the four-point function of free-field vertex operators:

$$
\left\langle\prod_{i=1}^{4} \mathcal{O}_{\beta_{i}}\left(z_{i}\right)\right\rangle_{\mathbb{C}} \rightarrow \prod_{\substack{i, j=1 \\ i<j}}^{4}\left|z_{i j}\right|^{4 \gamma_{i} \gamma_{j}}=\left\langle\prod_{j=1}^{4} e^{i \sqrt{2} \gamma_{j} \psi\left(z_{j}\right)}\right\rangle .
$$


This same limit should reduce the $n$-point function in the plane for all $n$ to the free-field case.

Interestingly, the correlators in the upper half-plane do not reduce to those of free fields in the same limit. To see this, note that the coefficient of the hypergeometric function in (3.13), $\Delta_{1}+\Delta_{2}-\Delta_{12}$, does not vanish in the limit described above. (This is in contrast to the coefficient of the hypergeometric functions in (5.9), see (5.13).) Since the two-point function of free-field vertex operators in the upper half-plane is simply a product of powers of distances between $z_{1}$ and $z_{2}$ and their images in the lower half-plane $\bar{z}_{1}$ and $\bar{z}_{2},(3.13)$ does not reduce to the free-field result. Apparently, the boundary condition on the real axis induces interactions between the bulk operators. The same limit considered here, but in domains conformally equivalent to a disc (which include the upper half-plane), is studied in detail in [18].

\section{Expansion in conformal blocks}

The four-point function of a conformal field theory contains information about the threepoint function coefficients, as well as the spectrum of primary operators. To obtain this data, one makes use of the operator algebra by performing a conformal block expansion.

By a global conformal transformation, one can always map three of the points appearing in the four-point function to fixed values. The remaining dependence is only on the cross-ratio (5.5)

$$
x=\frac{z_{12} z_{34}}{z_{13} z_{24}}
$$

and its conjugate $\bar{x}$. Each cross-ratio is invariant under global conformal transformations. Following the notation of [19] section 6.6.4, we set $z_{1}=\infty, z_{2}=1, z_{3}=x$ and $z_{4}=0$, and define

$$
G_{34}^{21}(x)=\lim _{z_{1} \rightarrow \infty} z_{1}^{2 \Delta_{1}} \bar{z}_{1}^{2 \bar{\Delta}_{1}}\left\langle\mathcal{O}_{\beta_{1}}\left(z_{1}\right) \mathcal{O}_{\beta_{2}}(1) \mathcal{O}_{\beta_{3}}(x) \mathcal{O}_{\beta_{4}}(0)\right\rangle_{\mathbb{C}}
$$

where $\bar{\Delta}_{1}=\Delta_{1}$ in our case (note that later on we will consider operators with spin, $\left.\Delta^{\left(p, p^{\prime}\right)} \neq \bar{\Delta}^{\left(p, p^{\prime}\right)}\right)$.

We can now proceed to expand the four-point function in Virasoro conformal blocks

$$
G_{34}^{21}(x)=\sum_{\mathcal{P}} C_{34}^{\mathcal{P}} C_{12}^{\mathcal{P}} \mathcal{F}_{34}^{21}(\mathcal{P} \mid x) \overline{\mathcal{F}}_{34}^{21}(\mathcal{P} \mid \bar{x})
$$

The sum over $\mathcal{P}$ runs over all primary operators in the theory, and the $C_{i j}^{\mathcal{P}}$ are the threepoint function coefficients of the operators labeled by $i, j$ with $\mathcal{P}$. Each $\mathcal{P}$ with a non-zero $C$ contributes a term consisting of a holomorphic function times an anti-holomorphic function of the cross-ratio. These functions - the Virasoro conformal blocks - depend only on $x$, the central charge $c$, and the conformal dimensions $\Delta_{i}, \Delta_{\mathcal{P}}$ of the five operators.

The conformal blocks are given perturbatively by a power series

$$
\mathcal{F}_{34}^{21}(\mathcal{P} \mid x)=x^{\Delta_{\mathcal{P}}-\Delta_{3}-\Delta_{4}} \sum_{K=0}^{\infty} \mathcal{F}_{K} x^{K},
$$


where the coefficients $F_{K}$ are determined by the Virasoro algebra. The first three terms are given by (see $[19,20])$

$$
\begin{aligned}
\mathcal{F}_{0} & =1 \\
\mathcal{F}_{1} & =\frac{\left(\Delta_{\mathcal{P}}+\Delta_{2}-\Delta_{1}\right)\left(\Delta_{\mathcal{P}}+\Delta_{3}-\Delta_{4}\right)}{2 \Delta_{\mathcal{P}}} \\
\mathcal{F}_{2} & =\frac{A+B}{C}
\end{aligned}
$$

with

$$
\begin{aligned}
A= & \left(\Delta_{\mathcal{P}}+\Delta_{2}-\Delta_{1}\right)\left(\Delta_{\mathcal{P}}+\Delta_{2}-\Delta_{1}+1\right) \\
& \times\left[\left(\Delta_{\mathcal{P}}+\Delta_{3}-\Delta_{4}\right)\left(\Delta_{\mathcal{P}}+\Delta_{3}-\Delta_{4}+1\right)\left(4 \Delta_{\mathcal{P}}+c / 2\right)-6 \Delta_{\mathcal{P}}\left(\Delta_{\mathcal{P}}+2 \Delta_{3}-\Delta_{4}\right)\right] \\
B= & 4 \Delta_{\mathcal{P}}\left(2 \Delta_{\mathcal{P}}+1\right)\left(4 \Delta_{\mathcal{P}}+c / 2\right)-36 \Delta_{\mathcal{P}}^{2} \\
C= & \left(\Delta_{\mathcal{P}}+2 \Delta_{2}-\Delta_{1}\right) \\
& \times\left[4 \Delta_{\mathcal{P}}\left(2 \Delta_{\mathcal{P}}+1\right)\left(\Delta_{\mathcal{P}}+2 \Delta_{3}-\Delta_{4}\right)-6 \Delta_{\mathcal{P}}\left(\Delta_{\mathcal{P}}+\Delta_{3}-\Delta_{4}\right)\left(\Delta_{\mathcal{P}}+\Delta_{3}-\Delta_{4}+1\right)\right] .
\end{aligned}
$$

We now take the limit in the four-point function (5.9) to obtain

$$
G_{34}^{21}(x)=|x|^{2\left(\Delta_{12}-\Delta_{3}-\Delta_{4}\right)}|1-x|^{2\left(\Delta_{14}-\Delta_{2}-\Delta_{3}\right)} \exp [2 \tilde{\Delta} A(x)],
$$

where $A(x)$ is given by (5.6) and

$$
\begin{aligned}
\tilde{\Delta} & =\Delta_{12}+\Delta_{13}+\Delta_{14}-\Delta_{1}-\Delta_{2}-\Delta_{3}-\Delta_{4}, \\
= & \frac{\lambda}{10}\left[-1+\cos \beta_{1}+\cos \beta_{2}+\cos \beta_{3}+\cos \beta_{4}\right. \\
& \left.\quad-\cos \left(\beta_{1}+\beta_{2}\right)-\cos \left(\beta_{1}+\beta_{3}\right)-\cos \left(\beta_{1}+\beta_{4}\right)\right] .
\end{aligned}
$$

Any consistent four-point function of scalar primary operators must obey the crossing relations:

$$
\begin{aligned}
G_{34}^{21}(x) & =G_{32}^{41}(1-x) \\
& =|x|^{-4 \Delta_{3}} G_{31}^{24}\left(\frac{1}{x}\right) .
\end{aligned}
$$

These relations follow from the invariance of (5.9) under exchange of any pair of indices, which we have already verified. However as a check, we can verify (6.9a) directly. By taking different limits of the four points we obtain

$$
\begin{aligned}
& G_{32}^{41}(x)=|x|^{2\left(\Delta_{14}-\Delta_{2}-\Delta_{3}\right)}|1-x|^{2\left(\Delta_{12}-\Delta_{3}-\Delta_{4}\right)} \exp [2 \tilde{\Delta} A(x)] \\
& G_{31}^{24}(x)=|x|^{2\left(\Delta_{13}-\Delta_{1}-\Delta_{3}\right)}|1-x|^{2\left(\Delta_{14}-\Delta_{2}-\Delta_{3}\right)} \exp [2 \tilde{\Delta} A(x)] .
\end{aligned}
$$

It is easy to see that (6.7), (6.10) and (6.11) indeed satisfy (6.9a) (again making use of the identity (5.10)). 


\subsection{Primary operator spectrum}

As explained above, the expansion of $G_{34}^{21}(x)$ around $x=0$ reveals the spectrum of dimensions of the primary operators that couple to the layering vertex operators. The hypergeometric functions appearing in $A(x)$ are regular as $x \rightarrow 0$. As a result, the leading power comes from the term $|x|^{2\left(\Delta_{12}-\Delta_{3}-\Delta_{4}\right)}$, where $\Delta_{12}=\Delta_{34}=\frac{\lambda}{10}\left(1-\cos \left(\beta_{3}+\beta_{4}\right)\right)$. Therefore, the lightest operator with non-zero three point function with $e^{i \beta_{3} N\left(z_{3}\right)} e^{i \beta_{4} N\left(z_{4}\right)}$ and $e^{i \beta_{1} N\left(z_{1}\right)} e^{i \beta_{2} N\left(z_{2}\right)}$ has dimension $\Delta_{12}=\Delta_{34}$, the dimension of the operator $e^{-i\left(\beta_{3}+\beta_{4}\right) N(z)}$. Furthermore, the three-point function coefficient is equal to 1. Presumably, this operator is indeed $e^{-i\left(\beta_{3}+\beta_{4}\right) N(z)}=e^{i\left(\beta_{1}+\beta_{2}\right) N(z)}$, although we cannot be certain as we do not have complete knowledge of all its three-point function coefficients.

The next term in the expansion of $G_{34}^{21}(x)$ comes from the $x^{1 / 3}{ }_{2} F_{1}(2 / 3,1 ; 4 / 3 ; x)=$ $x^{1 / 3}(1+\mathcal{O}(x))$ term. Since there are no other terms with the power $x^{1 / 3}$, there must be at least one primary operator with dimension $\Delta_{12}+1 / 3$. Similarly, expanding the exponential to quadratic order gives a term proportional to the square of the previous one, $x^{2 / 3}{ }_{2} F_{1}(2 / 3,1 ; 4 / 3 ; x)^{2} / 2$. This indicates the existence of a primary with dimension $\Delta_{12}+2 / 3$.

The question of whether there is a primary with $\Delta_{12}+3 / 3=\Delta_{12}+1$ is more subtle, because this power of $x$ also appears in the expansion of the $\Delta_{12}$ conformal block. To see that such an operator indeed exists, we could compute (the square of) its three-point function coefficient by subtracting the contribution from that of the $\Delta_{12}$ block, and see that the result is non-zero.

Let us now make this procedure systematic for the first few levels of operators. As noted before, it appears there exist operators of dimension $\left(\Delta^{p, p^{\prime}}, \bar{\Delta}^{p, p^{\prime}}\right)$ with

$$
\begin{aligned}
& \Delta^{\left(p, p^{\prime}\right)}=\Delta_{12}+\frac{p}{3}=\Delta_{34}+\frac{p}{3}=\frac{\lambda}{10}\left(1-\cos \left(\beta_{1}+\beta_{2}\right)\right)+\frac{p}{3} \\
& \bar{\Delta}^{\left(p, p^{\prime}\right)}=\frac{\lambda}{10}\left(1-\cos \left(\beta_{1}+\beta_{2}\right)\right)+\frac{p^{\prime}}{3}
\end{aligned}
$$

for some non-negative integers $p, p^{\prime}$ that couple to $e^{i \beta_{3} N\left(z_{3}\right)} e^{i \beta_{4} N\left(z_{4}\right)}$ and $e^{i \beta_{1} N\left(z_{1}\right)} e^{i \beta_{2} N\left(z_{2}\right)}$. We need to compare the expansion of

$$
G_{34}^{21}(x)=|x|^{-2\left(\Delta_{3}+\Delta_{4}\right)} \sum_{m, n=0}^{\infty} a_{m n} x^{m / 3} \bar{x}^{n / 3}
$$

to the conformal block expansion (6.3) in which we must allow the operators to have nonzero spin $\left(\Delta^{p, p^{\prime}}\right.$ may be different from $\left.\bar{\Delta}^{p, p^{\prime}}\right)$. To accommodate the previous results we write

$$
G_{34}^{21}(x)=|x|^{-2\left(\Delta_{3}+\Delta_{4}\right)} \sum_{\substack{p, p^{\prime}, i, j=0}}^{\infty} C_{34}^{\left(p, p^{\prime}\right)} C_{12}^{\left(p, p^{\prime}\right)} \mathcal{F}_{i}^{(p)} \overline{\mathcal{F}}_{j}^{\left(p^{\prime}\right)} x^{i+p / 3} \bar{x}^{j+p^{\prime} / 3},
$$

where we now sum over all non-negative integers $i, j, p, p^{\prime}$. Here $F_{i}^{(p)} \bar{F}_{j}^{\left(p^{\prime}\right)}$ denote the conformal block coefficients evaluated at (6.12).

By identifying the coefficients order by order in $x$ and $\bar{x}$ we can find the products of three-point coefficients $C_{34}^{\left(p, p^{\prime}\right)} C_{12}^{\left(p, p^{\prime}\right)}$. Every non-zero combination shows the existence of 


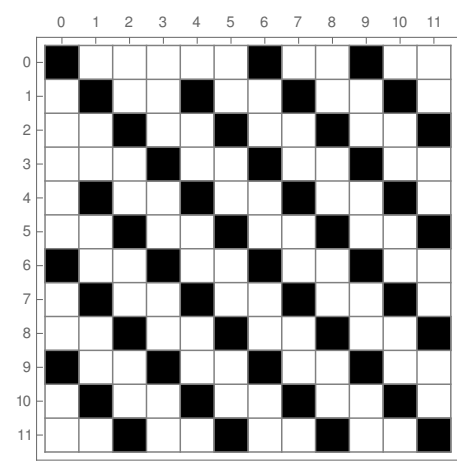

(a) Generic $\beta_{i}$.

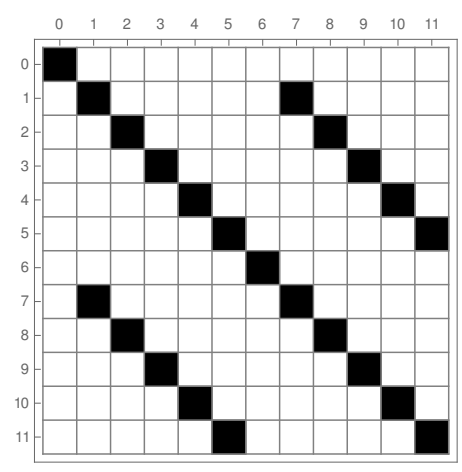

(b) $\beta_{1}=\beta_{2}=\beta_{3}=\beta_{4}=\pi$.

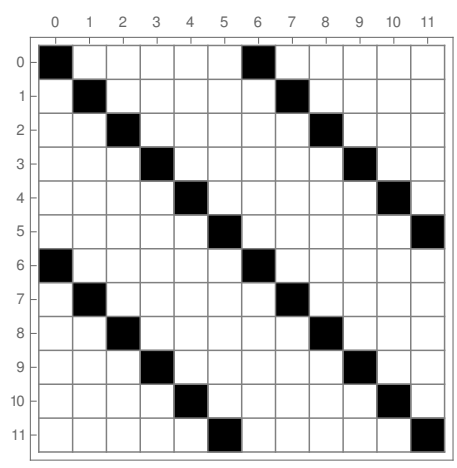

(c) $\beta_{1}=\beta_{2}=\beta_{3}=\beta_{4}=\frac{\pi}{2}$.

Figure 2. The non-zero products $C_{34}^{\left(p, p^{\prime}\right)} C_{12}^{\left(p, p^{\prime}\right)}$ are shown for different choices of $\beta_{i}$.

an operator with dimensions $\left(\Delta^{p, p^{\prime}}, \bar{\Delta}^{p, p^{\prime}}\right)$ in the operator spectrum to which two layering vertex operators fuse.

We use a code developed by Matt Headrick [20] to generate the conformal block coefficients up to third order, which allows us to consider terms up to order $\mathcal{O}\left(x^{11 / 3}\right)$. The non-zero three point coefficients are marked in figure 2 . We denote $\mu=\frac{2^{1 / 3} \pi^{2}}{3 \sqrt{3} \Gamma(1 / 6)^{2} \Gamma(4 / 3)^{2}}$. Below, we give the first few three-point function coefficients from different blocks. These grow very rapidly in complexity with increasing $p, p^{\prime}$.

$$
\begin{aligned}
& C_{34}^{(0,0)} C_{12}^{(0,0)}=1 \\
& C_{34}^{(1,1)} C_{12}^{(1,1)}=-\frac{48}{5} \mu \lambda \sin \frac{\beta_{1}}{2} \sin \frac{\beta_{2}}{2} \sin \frac{\beta_{3}}{2} \sin \frac{\beta_{1}+\beta_{2}+\beta_{3}}{2} \\
& C_{34}^{(2,2)} C_{12}^{(2,2)}=\frac{1}{2}\left(C_{34}^{(1,1)} C_{12}^{(1,1)}\right)^{2} \\
& C_{34}^{(3,3)} C_{12}^{(3,3)}=\frac{1}{3 !}\left(C_{34}^{(1,1)} C_{12}^{(1,1)}\right)^{3} .
\end{aligned}
$$

$C_{34}^{(4,4)} C_{12}^{(4,4)}$ and the following terms are very lengthy. The first few off-diagonal terms of the form $C^{(n, n+3)}=C^{(n+3, n)}$ are

$$
\begin{aligned}
& C_{34}^{(0,3)} C_{12}^{(0,3)}=0 \\
& C_{34}^{(1,4)} C_{12}^{(1,4)}=\frac{24 \mu \lambda^{2}}{5} \frac{\left(\cos \frac{\beta_{3}}{2}-\cos \left(\beta_{1}+\beta_{2}+\frac{3 \beta_{3}}{2}\right)\right) \sin \frac{\beta_{1}}{2} \sin \left(\frac{\beta_{1}-\beta_{2}}{2}\right) \sin \frac{\beta_{2}}{2} \sin \frac{\beta_{3}}{2}}{-10-3 \lambda\left(1-\cos \left(\beta_{1}+\beta_{2}\right)\right)} \\
& C_{34}^{(2,5)} C_{12}^{(2,5)}=\frac{1152 \mu^{2} \lambda^{3}}{25} \frac{\left(\cos \left(\beta_{1}+\beta_{3}\right)-\cos \left(\beta_{2}+\beta_{3}\right)\right)\left(\sin \frac{\beta_{1}}{2} \sin \frac{\beta_{2}}{2} \sin \frac{\beta_{3}}{2} \sin \left(\frac{\beta_{1}+\beta_{2}+\beta_{3}}{2}\right)\right)^{2}}{-20-3 \lambda\left(1-\cos \left(\beta_{1}+\beta_{2}\right)\right)} \\
& C_{34}^{(3,6)} C_{12}^{(3,6)}=-\frac{9216 \mu^{3} \lambda^{4}}{125} \frac{\left(\cos \left(\beta_{1}+\beta_{3}\right)-\cos \left(\beta_{2}+\beta_{3}\right)\right)\left(\sin \frac{\beta_{1}}{2} \sin \frac{\beta_{2}}{2} \sin \frac{\beta_{3}}{2} \sin \left(\frac{\beta_{1}+\beta_{2}+\beta_{3}}{2}\right)\right)^{3}}{-10-\lambda\left(1-\cos \left(\beta_{1}+\beta_{2}\right)\right)} .
\end{aligned}
$$


The first term of the form $C^{(n, n+6)}=C^{(n+6, n)}$ is

$$
\begin{aligned}
C_{34}^{(0,6)} C_{12}^{(0,6)}= & -\frac{\lambda^{2}}{200}\left[11+5\left(\cos \beta_{1}+\cos \beta_{2}-\cos \left(\beta_{1}+\beta_{2}\right)\right]\right. \\
& \times \sin \frac{\beta_{1}}{2} \sin \frac{\beta_{2}}{2} \sin \frac{\beta_{3}}{2}\left[6 \sin \frac{\beta_{1}+\beta_{2}-\beta_{3}}{2}+17 \sin \frac{\beta_{1}+\beta_{2}+\beta_{3}}{2}\right. \\
& \left.\quad+5 \sin \frac{3\left(\beta_{1}+\beta_{2}+\beta_{3}\right)}{2}-6 \sin \frac{3\left(\beta_{1}+\beta\right)+\beta_{3}}{2}+5 \sin \frac{\beta_{1}+\beta_{2}+3 \beta_{3}}{2}\right] \\
& \times\left[25+14 \lambda+\cos \left(\beta_{1}+\beta_{2}\right)\left(25-\lambda\left(18-4 \cos ^{2}\left(\beta_{1}+\beta_{2}\right)\right)\right)\right]^{-1} .
\end{aligned}
$$

We now analyze two special cases for which the three-point coefficients simplify considerably. Consider first the case $\beta_{1}=\beta_{2}=\beta_{3}=\beta_{4}=\pi$. We denote $C_{34}^{\left(p, p^{\prime}\right)}=C_{12}^{\left(p, p^{\prime}\right)}=C^{\left(p, p^{\prime}\right)}$. The first few diagonal terms $C^{(n, n)}$ are given for $0 \leq n \leq 6$ by

$$
\left(C^{(n, n)}\right)^{2}=\frac{1}{n !}\left(C^{(1,1)}\right)^{2 n}
$$

with

$$
\left(C^{(1,1)}\right)^{2}=\frac{48}{5} \lambda \mu
$$

The term with $n=7$ is

$$
\left(C^{(7,7)}\right)^{2}=\frac{768 \mu \lambda^{3}}{19140625}\left(\frac{125}{(7-15 \lambda)^{2}}+37158912 \mu^{6} \lambda^{4}\right)
$$

The first few off-diagonal terms are of the form $C^{(n, n+6)}=C^{(n+6, n)}$

$$
\begin{aligned}
& \left(C^{(6,0)}\right)^{2}=0 \\
& \left(C^{(7,1)}\right)^{2}=-\frac{192}{875} \frac{\lambda^{2} \mu}{15 \lambda-7} \\
& \left(C^{(8,2)}\right)^{2}=\frac{288}{4375} \frac{161 \lambda-50}{21 \lambda+2} \lambda^{2} \mu^{2} \\
& \left(C^{(9,3)}\right)^{2}=\frac{1536}{21875} \frac{59 \lambda-25}{\lambda+1} \lambda^{3} \mu^{3} .
\end{aligned}
$$

Now consider the case $\beta_{1}=\beta_{2}=\beta_{3}=\beta_{4}=\frac{\pi}{2}$. The diagonal terms for $0 \leq n \leq 5$ are

$$
\left(C^{(n, n)}\right)^{2}=\frac{1}{n !}\left(C^{(1,1)}\right)^{2 n}
$$

with

$$
\left(C^{(1,1)}\right)^{2}=-\frac{12}{5} \lambda \mu
$$

The $n=6$ term is

$$
\left(C^{(6,6)}\right)^{2}=\frac{\lambda^{2}}{1250000}\left(125+331776 \mu^{6} \lambda^{4}\right)
$$


The first few off-diagonal terms $C^{(n, n+6)}$ are

$$
\begin{aligned}
& \left(C^{(6,0)}\right)^{2}=-\frac{\lambda}{100} \\
& \left(C^{(7,1)}\right)^{2}=\frac{3 \lambda^{2} \mu}{1750} \frac{2268 \lambda^{2}+5835 \lambda-1450}{162 \lambda^{2}+390 \lambda-175} \\
& \left(C^{(8,2)}\right)^{2}=-\frac{9 \lambda^{2} \mu^{2}}{4375} \frac{1134 \lambda^{3}+5835 \lambda^{2}+2350 \lambda+1250}{81 \lambda^{2}+390 \lambda+25} \\
& \left(C^{(9,3)}\right)^{2}=\frac{36 \lambda^{3} \mu^{3}}{21875} \frac{252 \lambda^{3}+1945 \lambda^{2}+2050 \lambda+1250}{18 \lambda^{2}+130 \lambda+75} .
\end{aligned}
$$

\subsection{Interpretation}

We leave the physical interpretation of these new primaries to future work. A hint is provided by [21], which considers the $O(n)$ model as $n \rightarrow 0$. There the four-point function is essentially the $\log$ of the one considered here for $\beta_{i}=\pi$, and only a finite number of primaries appear in the fusion products. The primary corresponding to $\left(p=1, p^{\prime}=1\right)$ in our notation has dimension $\left(\frac{1}{3}, \frac{1}{3}\right)$ when $\beta_{i}=\pi$, and is identified as the leading order energy density operator of the $O(n)$ model (in the limit $n \rightarrow 0$ ).

One important caveat to our results in this section is that the three-point function coefficients obtained from the conformal block expansion do not entirely determine the spectrum of primaries. Clearly, there could be primaries in the theory with vanishing three-point coefficients with the layering vertex operators, and these would be invisible to us. A more subtle issue can also arise in the other direction if there are multiple operators with the same conformal dimensions that couple to the vertex operators. In that case one can only determine the sum of the squares of the three-point coefficients. Since these squared coefficients can evidently be negative, there could be cancellations. Therefore it is logically possible we are missing some operators in the theory that couple to the vertex operators, as there could be multiple degenerate primaries that couple with three-point coefficients with squares that sum to zero.

\subsection{Null descendant states}

Some of the three-point function coefficients we have calculated diverge at special values of $\lambda, \beta_{i}$. For instance, with all $\beta_{i}=\pi$ we have

$$
\left(C^{(1,7)}\right)^{2}=\left(C^{(7,1)}\right)^{2}=-\frac{192}{875} \frac{\lambda^{2} \mu}{15 \lambda-7} .
$$

This coefficient diverges when $\lambda=7 / 15$, or $c=14 / 15$. One expects CFTs with $c<1$ to contain null descendants of primaries with certain conformal dimensions. Indeed, at $c=14 / 15$ the Kac determinant vanishes at second level for a primary with dimension $\Delta=1 / 3\left(h_{2,1}\right.$ in standard notation, see for instance [19]). Vanishing at second level means a state with dimension $1 / 3$ should become null. When this happens the corresponding three point coefficient diverges, because the norm of the state appears in the denominator. The dimension of the operator corresponding to $C^{(1,7)}$ is indeed $\Delta^{(1,7)}=(\lambda / 10)\left(1-\cos \left(\beta_{1}+\right.\right.$ $\left.\left.\beta_{2}\right)\right)+p / 3=1 / 3$ for $\beta_{i}=\pi$ and $p=1$, as expected from this argument. ${ }^{3}$

\footnotetext{
${ }^{3}$ We thank Alex Maloney and Liam Fitzpatrick for discussions on this point.
} 
The four-point function (5.9) is an analytic function of all its parameters and arguments. In particular, the conformal block expansion (6.14) remains finite even when three-point coefficients diverge due to a null state. This occurs because the conformal blocks $\mathcal{F}_{i}^{(p)}$ vanish so that the product $C_{34}^{\left(p, p^{\prime}\right)} C_{12}^{\left(p, p^{\prime}\right)} \mathcal{F}_{i}^{(p)} \mathcal{F}_{j}^{\left(p^{\prime}\right)}$ remains finite, which is the expected behavior in general for the conformal block expansion of correlation functions of primary operators with null descendant states.

\section{Outlook}

Our results for the correlation functions raise many interesting questions. First, it is possible that we can extend our techniques to compute $n$-point correlation functions for arbitrary $n$. This would provide new results for the winding probabilities of Brownian loops. The spectrum of new primary operators we discovered needs investigation, as we do not know how to identify these operators either in terms of a previously known CFT, or in terms of the BLS.

Another interesting direction is to generalize the random variables assigned to the loops. Here we considered the layering operator and assigned a random \pm 1 to each loop. In ongoing work to appear soon, two of us (Foit and Kleban) have considered more general distributions of random weights. This gives rise to an infinite class of new conformally invariant systems for which we can compute exact four-point functions that depend on additional continuous parameters characterizing the distribution of weights.

\section{Acknowledgments}

We would like to thank Liam Fitzpatrick, Ben Freivogel, Gaston Giribet, Matthew Headrick, Alex Maloney, and Massimo Porrati for useful discussions, and an anonymous referee for useful and constructive comments. The work of V. F. is supported by the James Arthur Graduate Award. The work of M. K. is supported by the NSF through the grant PHY-1820814.

\section{A Full-plane limits}

In this appendix, we derive the results of section 4 by taking limits of our results from the upper half-plane.

We consider the two-point function first and apply charge conservation, which implies $\Delta_{12}=0$. Taking

$$
\zeta_{1}(t)=x_{1}+i\left(y_{1}+t\right), \quad \zeta_{2}(t)=x_{2}+i\left(y_{2}+t\right)
$$

with $t \geq 0$ and $z_{1}=x_{1}+i y_{1}, z_{2}=x_{2}+i y_{2}$, we can express the two-point function in the complex plane as the limit of two-point functions in the upper half-plane, as follows:

$$
\left\langle e^{i \beta_{1} N\left(z_{1}\right)} e^{i \beta_{2} N\left(z_{2}\right)}\right\rangle_{\mathbb{C}}=\lim _{t \rightarrow \infty}\left\langle e^{i \beta_{1} N\left(\zeta_{1}(t)\right)} e^{i \beta_{2} N\left(\zeta_{2}(t)\right)}\right\rangle_{\mathbb{H}} .
$$


To see this, note that

$$
\begin{aligned}
\left\langle e^{i \beta_{1} N\left(\zeta_{1}(t)\right)} e^{i \beta_{2} N\left(\zeta_{2}(t)\right)}\right\rangle_{\mathbb{H}} & =\left\langle e^{i \beta_{1} N\left(z_{1}\right)} e^{i \beta_{2} N\left(z_{2}\right)}\right\rangle_{\mathbb{H}_{t}} \\
& =e^{-\lambda \alpha_{\mathbb{H}_{t}}\left(z_{1} \mid z_{2}\right)\left(1-\cos \beta_{1}\right)} e^{-\lambda \alpha_{\mathbb{H}_{t}}\left(z_{2} \mid z_{1}\right)\left(1-\cos \beta_{2}\right)},
\end{aligned}
$$

where $\mathbb{H}_{t}$ is the half-plane $\{(x, y): y \geq-t\}$. The weights $\alpha_{\mathbb{H}_{t}}\left(z_{j} \mid z_{k}\right)$, for unequal $j, k=1,2$, are increasing in $t$ and bounded above by $\alpha\left(z_{j} \mid z_{k}\right)$, which is finite by thinness of the Brownian loop measure $\mu^{\text {loop }}[22]$; this implies that they have a finite limit as $t \rightarrow \infty$. Moreover

$$
\alpha\left(z_{j} \mid z_{k}\right)-\alpha_{\mathbb{H}_{t}}\left(z_{j} \mid z_{k}\right) \leq \mu^{\operatorname{loop}}\left(\gamma: \operatorname{diam}(\gamma) \geq t, \gamma \text { intersect } \overline{z_{1} z_{2}}\right) \rightarrow 0
$$

as $t \rightarrow \infty$, where $\overline{z_{1} z_{2}}$ is the segment connecting $z_{1}$ and $z_{2}$, again by thinness. This shows that $\lim _{t \rightarrow \infty} \alpha_{\mathbb{H}_{t}}\left(z_{i} \mid z_{j}\right)=\alpha\left(z_{i} \mid z_{j}\right)$ and proves (A.2).

We are interested in calculating

$$
\left\langle\mathcal{O}_{\beta_{1}}\left(z_{1}\right) \mathcal{O}_{\beta_{2}}\left(z_{2}\right)\right\rangle_{\mathbb{C}}=\lim _{\delta \rightarrow 0} \frac{\left\langle e^{i \beta_{1} N\left(z_{1}\right)} e^{i \beta_{2} N\left(z_{2}\right)}\right\rangle_{\mathbb{C}}}{\left(2 \delta e^{-\frac{\pi}{\sqrt{3}}-5 \hat{\alpha}}\right)^{2\left(\Delta_{1}+\Delta_{2}\right)}}=\lim _{\delta \rightarrow 0} \lim _{t \rightarrow \infty} \frac{\left\langle e^{i \beta_{1} N\left(\zeta_{1}(t)\right)} e^{i \beta_{2} N\left(\zeta_{2}(t)\right)}\right\rangle_{\mathbb{H}}}{\left(2 \delta e^{-\frac{\pi}{\sqrt{3}}-5 \hat{\alpha}}\right)^{2\left(\Delta_{1}+\Delta_{2}\right)}}
$$

For any $\delta<\left|z_{1}-z_{2}\right|$, using Lemma A1 of [6] and letting $\alpha_{\left|z_{i}-z_{j}\right|, \mathbb{H}_{t}}\left(z_{i} \mid z_{j}\right)$ denote the weight of all loops of diameter at least $\left|z_{i}-z_{j}\right|$ contained in $\mathbb{H}_{t}$ and winding around $z_{i}$ but not $z_{j}$, we have that

$$
\alpha_{\mathbb{H}_{t}}\left(z_{1} \mid z_{2}\right)=\frac{1}{5} \log \frac{\left|z_{1}-z_{2}\right|}{\delta}+\alpha_{\left|z_{1}-z_{2}\right|, \mathbb{H}_{t}}\left(z_{1} \mid z_{2}\right),
$$

which allows us to write

$$
\begin{aligned}
\delta^{-2\left(\Delta_{1}+\Delta_{2}\right)}\left\langle e^{i \beta_{1} N\left(\zeta_{1}(t)\right)} e^{i \beta_{2} N\left(\zeta_{2}(t)\right)}\right\rangle_{\mathbb{H}} & =\delta^{-2\left(\Delta_{1}+\Delta_{2}\right)}\left\langle e^{i \beta_{1} N\left(z_{1}\right)} e^{i \beta_{2} N\left(z_{2}\right)}\right\rangle_{\mathbb{H}_{t}} \\
& =e^{-\lambda \alpha_{\mathbb{H}_{t}}\left(z_{1} \mid z_{2}\right)\left(1-\cos \beta_{1}\right)} e^{-\lambda \alpha_{\mathbb{H}_{t}}\left(z_{2} \mid z_{1}\right)\left(1-\cos \beta_{2}\right)} \\
& =\frac{e^{-10 \Delta_{1} \alpha_{\left|z_{1}-z_{2}\right|, \mathbb{H}_{t}}\left(z_{1} \mid z_{2}\right)} e^{-10 \Delta_{2} \alpha_{\left|z_{1}-z_{2}\right|, \mathbb{H}_{t}}\left(z_{2} \mid z_{1}\right)}}{\left|z_{1}-z_{2}\right|^{2\left(\Delta_{1}+\Delta_{2}\right)}},
\end{aligned}
$$

which is independent of $\delta$. Because of this, we can exchange the limits in (A.5) and write

$$
\begin{aligned}
\left\langle\mathcal{O}_{\beta_{1}}\left(z_{1}\right) \mathcal{O}_{\beta_{2}}\left(z_{2}\right)\right\rangle_{\mathbb{C}} & =\lim _{t \rightarrow \infty} \lim _{\delta \rightarrow 0} \frac{\left\langle e^{i \beta_{1} N\left(\zeta_{1}(t)\right)} e^{i \beta_{2} N\left(\zeta_{2}(t)\right)}\right\rangle_{\mathbb{H}}}{\left(2 \delta e^{-\frac{\pi}{\sqrt{3}}-5 \hat{\alpha}}\right)^{2\left(\Delta_{1}+\Delta_{2}\right)}} \\
& =\lim _{t \rightarrow \infty}\left\langle\mathcal{O}_{\beta_{1}}\left(\zeta_{1}(t)\right) \mathcal{O}_{\beta_{2}}\left(\zeta_{2}(t)\right)\right\rangle_{\mathbb{H}} .
\end{aligned}
$$

Notice also that

$$
\begin{aligned}
& \lim _{t \rightarrow \infty} \frac{\zeta_{1}(t)-\bar{\zeta}_{1}(t)}{\zeta_{1}(t)-\bar{\zeta}_{2}(t)}=\lim _{t \rightarrow \infty} \frac{2 i\left(y_{1}+t\right)}{\left(x_{1}-x_{2}\right)+i\left(y_{1}+y_{2}+2 t\right)}=1, \\
& \lim _{t \rightarrow \infty} \frac{\zeta_{2}(t)-\bar{\zeta}_{2}(t)}{\zeta_{1}(t)-\bar{\zeta}_{2}(t)}=\lim _{t \rightarrow \infty} \frac{2 i\left(y_{2}+t\right)}{\left(x_{1}-x_{2}\right)+i\left(y_{1}+y_{2}+2 t\right)}=1 .
\end{aligned}
$$


It follows that

$$
\lim _{t \rightarrow \infty} \eta=0, \quad \lim _{t \rightarrow \infty} \sigma=0, \quad \lim _{t \rightarrow \infty} \frac{\sigma}{\eta}=1
$$

and

$$
\lim _{\eta \rightarrow 0} \eta_{3} F_{2}\left(1,1, \frac{4}{3} ; 2, \frac{5}{3} ; \eta\right)=\lim _{\eta \rightarrow 0} \eta_{2} F_{1}\left(1, \frac{2}{3} ; \frac{4}{3}, \eta\right)=0 .
$$

From the equality of (3.3) and (3.4) we have that

$$
\lim _{t \rightarrow \infty}(1-\sigma)_{3} F_{2}\left(1,1, \frac{4}{3} ; 2, \frac{5}{3} ; 1-\sigma\right)=\frac{2 \pi}{\sqrt{3}} .
$$

Applying charge conservation $\beta_{1}+\beta_{2}=2 \pi \mathbb{Z}$, from (3.13), (A.8) and (4.2) we have that

$$
\begin{aligned}
\left\langle\tilde{\mathcal{O}}_{\beta_{1}}\left(z_{1}\right) \tilde{\mathcal{O}}_{\beta_{2}}\left(z_{2}\right)\right\rangle_{\mathbb{C}}= & \lim _{t \rightarrow \infty}\left\langle\tilde{\mathcal{O}}_{\beta_{1}}\left(\zeta_{1}(t)\right) \tilde{\mathcal{O}}_{\beta_{2}}\left(\zeta_{2}(t)\right)\right\rangle_{\mathbb{H}} \\
= & \lim _{t \rightarrow \infty}\left|\zeta_{1}(t)-\zeta_{2}(t)\right|^{-2\left(\Delta_{1}+\Delta_{2}\right)}\left|\zeta_{1}(t)-\bar{\zeta}_{2}(t)\right|^{2\left(\Delta_{1}+\Delta_{2}\right)} \\
& \times\left|\zeta_{1}(t)-\bar{\zeta}_{1}(t)\right|^{-2 \Delta_{1}}\left|\zeta_{2}(t)-\bar{\zeta}_{2}(t)\right|^{-2 \Delta_{2}} \\
& \times \exp \left[-\left(\Delta_{1}+\Delta_{2}\right)(1-\sigma)_{3} F_{2}\left(1,1, \frac{4}{3} ; 2, \frac{5}{3} ; 1-\sigma\right)\right] \\
= & \left(e^{\frac{\pi}{\sqrt{3}}}\right)^{-4 \Delta_{1}}\left|z_{1}-z_{2}\right|^{-4 \Delta_{1}}
\end{aligned}
$$

where we used the fact that $\Delta_{1}=\Delta_{2}$. This gives an expression in terms of $\hat{\alpha}$ for the constant appearing in the full-plane two-point function in [6]; with the normalization used in [6] (see the Summary and Results section, below (2.3)) the constant $C_{2}$ defined there is

$$
C_{2}=\left(2 e^{-\frac{\pi}{\sqrt{3}}-5 \hat{\alpha}}\right)^{2\left(\Delta_{1}+\Delta_{2}\right)}=\left(2 e^{-\frac{\pi}{\sqrt{3}}-5 \hat{\alpha}}\right)^{4 \Delta_{1}} .
$$

Absorbing the constants $\left(2 e^{-\frac{\pi}{\sqrt{3}}-5 \hat{\alpha}}\right)^{2 \Delta_{j}}$ into the definition of $\mathcal{O}_{\beta_{j}}$ (see (4.2)) gives the canonically normalized two-point function in the plane:

$$
\left\langle\mathcal{O}_{\beta_{1}}\left(z_{1}\right) \mathcal{O}_{\beta_{2}}\left(z_{2}\right)\right\rangle_{\mathbb{C}}=\left|z_{1}-z_{2}\right|^{-4 \Delta_{1}} .
$$

It turns out that we can also compute the three-point functions in the full plane using only the Brownian loop weights of the collections of loops encircling one and two points in the upper half-plane. Given $z_{i}, z_{j}, z_{k} \in \mathbb{C}$, for distinct $i, j, k \in\{1,2,3\}$ and $\delta \leq \min _{i j}\left|z_{i}-z_{j}\right|$, we have the six relations

$$
\begin{aligned}
\alpha_{\mathbb{H}}\left(z_{i}\right) & =\alpha_{\mathbb{H}}\left(z_{i} \mid z_{j}, z_{k}\right)+\alpha_{\mathbb{H}}\left(z_{i}, z_{j} \mid z_{k}\right)+\alpha_{\mathbb{H}}\left(z_{i}, z_{k} \mid z_{j}\right)+\alpha_{\mathbb{H}}\left(z_{1}, z_{2}, z_{3}\right) \\
\alpha_{\mathbb{H}}\left(z_{i}, z_{j}\right) & =\alpha_{\mathbb{H}}\left(z_{i}, z_{j} \mid z_{k}\right)+\alpha_{\mathbb{H}}\left(z_{1}, z_{2}, z_{3}\right)
\end{aligned}
$$

which give

$$
\begin{aligned}
& \alpha_{\mathbb{H}}\left(z_{i}, z_{j} \mid z_{k}\right)=\alpha_{\mathbb{H}}\left(z_{i}, z_{j}\right)-\alpha_{\mathbb{H}}\left(z_{1}, z_{2}, z_{3}\right) \\
& \alpha_{\mathbb{H}}\left(z_{i} \mid z_{j}, z_{k}\right)=\alpha_{\mathbb{H}}\left(z_{i}\right)-\alpha_{\mathbb{H}}\left(z_{i}, z_{j}\right)-\alpha_{\mathbb{H}}\left(z_{i}, z_{k}\right)+\alpha_{\mathbb{H}}\left(z_{1}, z_{2}, z_{3}\right) .
\end{aligned}
$$


It follows from (3.1) that

$$
\begin{gathered}
\left\langle e^{i \beta_{1} N\left(z_{1}\right)} e^{i \beta_{2} N\left(z_{2}\right)} e^{i \beta_{3} N\left(z_{3}\right)}\right\rangle_{\mathbb{H}} \\
=\exp \left[-\lambda\left(\sum_{j=1}^{3}\left(1-\cos \beta_{j}\right) \alpha_{\mathbb{H}}\left(z_{j} \mid z_{i}, z_{k}\right)+\sum_{\substack{j, k=1 \\
j<k}}^{3}\left(1-\cos \left(\beta_{j}+\beta_{k}\right)\right) \alpha_{\mathbb{H}}\left(z_{j}, z_{k} \mid z_{i}\right)\right.\right. \\
\left.\left.\quad+\left(1-\cos \left(\beta_{1}+\beta_{2}+\beta_{3}\right)\right) \alpha_{\mathbb{H}}\left(z_{1}, z_{2}, z_{3}\right)\right)\right] \\
=\exp \left[-\lambda\left(\sum_{\substack{j=1 \\
3}}^{3}\left(1-\cos \beta_{j}\right) \alpha_{\delta, \mathbb{H}}\left(z_{j}\right)\right.\right. \\
\quad+\sum_{\substack{j, k=1 \\
j<k}}^{3}\left(-\left(1-\cos \beta_{j}\right)-\left(1-\cos \beta_{k}\right)+\left(1-\cos \left(\beta_{j}+\beta_{k}\right)\right)\right) \alpha_{\mathbb{H}}\left(z_{j}, z_{k}\right) \\
\left.\left.+\left(\sum_{j=1}^{3}\left(1-\cos \beta_{j}\right)-\sum_{\substack{j, k=1 \\
j<k}}^{3}\left(1-\cos \left(\beta_{j}+\beta_{k}\right)\right)+1-\cos \left(\beta_{1}+\beta_{2}+\beta_{3}\right)\right) \alpha_{\mathbb{H}}\left(z_{1}, z_{2}, z_{3}\right)\right)\right] .
\end{gathered}
$$

Charge conservation, $\beta_{1}+\beta_{2}+\beta_{3}=2 \pi \mathbb{Z}$, implies that $\cos \left(\beta_{i}+\beta_{j}\right)=\cos \left(\beta_{k}\right)$, and hence the coefficient of $\alpha_{\mathbb{H}}\left(z_{1}, z_{2}, z_{3}\right)$ is identically zero. Using (3.7) and letting $\sigma_{j k}=\frac{\left|z_{j}-z_{k}\right|^{2}}{\left|z_{j}-\bar{z}_{k}\right|^{2}}$, this gives

$$
\begin{aligned}
& \left\langle e^{i \beta_{1} N\left(z_{1}\right)} e^{i \beta_{2} N\left(z_{2}\right)} e^{i \beta_{3} N\left(z_{3}\right)}\right\rangle_{\mathbb{H}} \\
& =\left(2 \delta e^{-5 \hat{\alpha}}\right)^{2\left(\Delta_{1}+\Delta_{2}+\Delta_{3}\right)} \prod_{i=1}^{3}\left|z_{i}-\bar{z}_{i}\right|^{-2 \Delta_{i}} \prod_{\substack{j, k=1 \\
j<k}}^{3}\left(\left|z_{j}-z_{k}\right|^{-2\left(\Delta_{j}+\Delta_{k}-\Delta_{j k}\right)}\left|z_{j}-\bar{z}_{k}\right|^{2\left(\Delta_{j}+\Delta_{k}-\Delta_{j k}\right)}\right. \\
& \left.\quad \times \exp \left[-\lambda\left(\Delta_{j}+\Delta_{k}-\Delta_{j k}\right)\left(1-\sigma_{j k}\right)_{3} F_{2}\left(1,1, \frac{4}{3} ; 2, \frac{5}{3} ; 1-\sigma_{j k}\right)\right]\right)
\end{aligned}
$$

The three-point function in the full plane can be obtained as a limit of three-point functions in the upper half-plane, as in the case of the two-point function treated above (see (A.8)). Using charge conservation and the full-plane canonical normalization factor, and letting 
$\zeta_{j}(t)=z_{j}+i t$, this leads to

$$
\begin{aligned}
& \left\langle\mathcal{O}_{\beta_{1}}\left(z_{1}\right) \mathcal{O}_{\beta_{2}}\left(z_{2}\right) \mathcal{O}_{\beta_{3}}\left(z_{3}\right)\right\rangle_{\mathbb{C}} \\
& =\lim _{\delta \rightarrow 0} \lim _{t \rightarrow \infty}\left(2 \delta e^{-5 \hat{\alpha}-\frac{\pi}{\sqrt{3}}}\right)^{-2 \sum_{j=1}^{3} \Delta_{j}}\left\langle e^{i \beta_{1} N\left(\zeta_{1}(t)\right)} e^{i \beta_{2} N\left(\zeta_{2}(t)\right)} e^{i \beta_{3} N\left(\zeta_{3}(t)\right)}\right\rangle_{\mathbb{H}} \\
& =\lim _{t \rightarrow \infty} \lim _{\delta \rightarrow 0}\left(2 \delta e^{-5 \hat{\alpha}-\frac{\pi}{\sqrt{3}}}\right)^{-2 \sum_{j=1}^{3} \Delta_{j}}\left\langle e^{i \beta_{1} N\left(\zeta_{1}(t)\right)} e^{i \beta_{2} N\left(\zeta_{2}(t)\right)} e^{i \beta_{3} N\left(\zeta_{3}(t)\right)}\right\rangle_{\mathbb{H}} \\
& =\lim _{t \rightarrow \infty}\left(e^{-\frac{\pi}{\sqrt{3}}}\right)^{-2 \sum_{j=1}^{3} \Delta_{j}} \prod_{i=1}^{3}\left|\zeta_{i}(t)-\bar{\zeta}_{i}(t)\right|^{-2 \Delta_{i}} \\
& \quad \times \prod_{j, k=1}^{3}\left(\left|\zeta_{j}(t)-\zeta_{k}(t)\right|^{-2\left(\Delta_{j}+\Delta_{k}-\Delta_{j k}\right)}\left|\zeta_{j}(t)-\bar{\zeta}_{k}(t)\right|^{2\left(\Delta_{j}+\Delta_{k}-\Delta_{j k}\right)}\right. \\
& \left.\quad \times \exp \left[-\left(\Delta_{j}+\Delta_{k}-\Delta_{j k}\right)(1-\sigma)_{3} F_{2}\left(1,1, \frac{4}{3} ; 2, \frac{5}{3} ; 1-\sigma\right)\right]\right) \\
& =\left|z_{1}-z_{2}\right|^{-2\left(\Delta_{1}+\Delta_{2}-\Delta_{12}\right)}\left|z_{1}-z_{3}\right|^{-2\left(\Delta_{1}+\Delta_{3}-\Delta_{13}\right)}\left|z_{2}-z_{3}\right|^{-2\left(\Delta_{2}+\Delta_{3}-\Delta_{23}\right)} \\
& =\left|z_{1}-z_{2}\right|^{-2\left(\Delta_{1}+\Delta_{2}-\Delta_{3}\right)}\left|z_{1}-z_{3}\right|^{-2\left(\Delta_{1}+\Delta_{3}-\Delta_{2}\right)}\left|z_{2}-z_{3}\right|^{-2\left(\Delta_{2}+\Delta_{3}-\Delta_{1}\right)},
\end{aligned}
$$

where we have used (A.12) to compute the $t \rightarrow \infty$ limit. Surprisingly, the overall coefficient of the three-point function is simply 1 , and does not depend on the $\beta_{i}$. (This was also the case for the three-point functions in the disk model of [11]).

From (A.20) we obtain an expression in terms of $\hat{\alpha}$ for the constant appearing in the three-point function in [6]; with the normalization used there (see the Summary and Results section, below (2.3))

$$
C_{3}=\left(2 e^{-\frac{\pi}{\sqrt{3}}-5 \hat{\alpha}}\right)^{2\left(\Delta_{1}+\Delta_{2}+\Delta_{3}\right)}
$$

\section{B Weights of loops covering one point and not a second}

With an ultraviolet cutoff $\delta$, the weight $\alpha\left(z_{1} \mid z_{2}\right)$ of loops in the plane that encircle $z_{1}$ but not $z_{2}$ is finite as a consequence of the thinness of the loop soup (see [22]). Starting from (3.10) and (3.4), one can derive an explicit expression for $\alpha\left(z_{1} \mid z_{2}\right)$ in terms of $\hat{\alpha}$, as follows. For two points $z_{1}, z_{2} \in \mathbb{C}, \delta \leq\left|z_{1}-z_{2}\right|$, and $z_{1}(t), z_{2}(t)$ as in appendix A, we have

$$
\begin{aligned}
\alpha\left(z_{1} \mid z_{2}\right)= & \alpha\left(z_{2} \mid z_{1}\right)=\lim _{t \rightarrow \infty} \alpha_{\mathbb{H}}\left(\zeta_{1}(t) \mid \zeta_{2}(t)\right) \\
= & \lim _{t \rightarrow \infty}\left[-\alpha_{\mathbb{H}}\left(\zeta_{1}(t), \zeta_{2}(t)\right)+\frac{1}{5} \log \left|\zeta_{1}(t)-\overline{\zeta_{1}(t)}\right|-\frac{1}{5} \log (2 \delta)+\hat{\alpha}\right] \\
= & \frac{1}{5} \log \left|z_{1}-z_{2}\right|-\frac{1}{5} \log (2 \delta)+\hat{\alpha} \\
& +\frac{1}{5} \lim _{t \rightarrow \infty}\left[\log \frac{\left|\zeta_{1}(t)-\overline{\zeta_{1}(t)}\right|}{\left|\zeta_{1}(t)-\overline{\zeta_{2}(t)}\right|}+\frac{1}{2}(1-\sigma)_{3} F_{2}\left(1,1, \frac{4}{3} ; 2, \frac{5}{3} ; 1-\sigma\right)\right] \\
= & \frac{1}{5} \log \left|z_{1}-z_{2}\right|+Q,
\end{aligned}
$$


where we defined the constant

$$
Q=\frac{\pi}{5 \sqrt{3}}-\frac{1}{5} \log (2 \delta)+\hat{\alpha}
$$

and again $\hat{\alpha}$ is the weight of loops in the upper half-plane with a diameter larger than 1 and that encircle $z=i$. Note that the analogous calculation of $\alpha\left(z_{1} \mid z_{2}, z_{3}\right)$ from (A.17) is not possible as $\alpha\left(z_{1}, z_{2}, z_{3}\right)$ is not known.

A similar computation, this time combining (3.10) with (3.3), provides an explicit expression for the linear term of the $O(n)$ expansion in [9], namely

$$
\begin{aligned}
\alpha_{\mathbb{H}}\left(z_{1} \mid z_{2}\right)+\alpha_{\mathbb{H}}\left(z_{2} \mid z_{1}\right) \\
=-2 \alpha_{\mathbb{H}}\left(z_{1}, z_{2}\right)+\frac{1}{5} \log \left(\left|z_{1}-\bar{z}_{1}\right|\left|z_{2}-\bar{z}_{2}\right|\right)-\frac{2}{5} \log (2 \delta)+2 \hat{\alpha} \\
=\frac{2 \pi}{5 \sqrt{3}}+\frac{1}{5} \eta_{3} F_{2}\left(1,1, \frac{4}{3} ; 2, \frac{5}{3} ; \eta\right)+\frac{1}{5} \log (\eta(\eta-1))-\frac{2 \Gamma\left(\frac{2}{3}\right)^{2}}{5 \Gamma\left(\frac{4}{3}\right)}(\eta(\eta-1))^{\frac{1}{3}}{ }_{2} F_{1}\left(1, \frac{2}{3} ; \frac{4}{3} ; \eta\right) \\
\quad+\frac{1}{5} \log \left(\left|z_{1}-\bar{z}_{1}\right|\left|z_{2}-\bar{z}_{2}\right|\right)-\frac{2}{5} \log (2 \delta)+2 \hat{\alpha} \\
=-\frac{1}{5}\left[-\eta_{3} F_{2}\left(1,1, \frac{4}{3} ; 2, \frac{5}{3} ; \eta\right)+\frac{2 \Gamma\left(\frac{2}{3}\right)^{2}}{\Gamma\left(\frac{4}{3}\right)}(\eta(\eta-1))^{\frac{1}{3}}{ }_{2} F_{1}\left(1, \frac{2}{3} ; \frac{4}{3} ; \eta\right)\right. \\
\left.\quad-\log \left(\eta\left(z_{1}-\bar{z}_{2}\right)\left(z_{2}-\bar{z}_{1}\right)\right)\right]+2 Q .
\end{aligned}
$$

This coincides with the expression in square brackets at the end of p. 12998 of [9] multiplied by $-3 \pi / 5$, which confirms the validity of the $O(n)$ expansion used in [9], at least in the case of the two-point function. While we cannot rigorously verify the validity of the expansion in the crucial case of the four-point function, the calculation above provides additional evidence in favor of the general validity of the $O(n)$ expansion and $n \rightarrow 0$ limit.

Open Access. This article is distributed under the terms of the Creative Commons Attribution License (CC-BY 4.0), which permits any use, distribution and reproduction in any medium, provided the original author(s) and source are credited.

\section{References}

[1] G.F. Lawler and W. Werner, The Brownian Loop Soup, Probabil. Theor. Relat. Fields 128 (2004) 565.

[2] W. Werner, The conformally invariant measure on self-avoiding loops, J. Amer. Math. Soc. 21 (2007) 137.

[3] S. Sheffield, Exploration trees and conformal loop ensembles, Duke Math. J. 147 (2009) 79.

[4] S. Sheffield and W. Werner, Conformal loop ensembles: the markovian characterization and the loop-soup construction, Ann. Math. 176 (2012) 1827 [arXiv:1006.2374].

[5] S. Benoist and C. Hongler, The scaling limit of critical Ising interfaces is $\mathrm{CLE}_{3}$, Ann. Probab. 47 (2019) 2049. 
[6] F. Camia, A. Gandolfi and M. Kleban, Conformal correlation functions in the Brownian Loop Soup, Nucl. Phys. B 902 (2016) 483 [arXiv:1501.05945] [INSPIRE].

[7] V.F. Foit and M. Kleban, New recipes for Brownian Loop Soups, arXiv:2007.01869 [INSPIRE].

[8] Y. Han, Y. Wang and M. Zinsmeister, On the brownian loop measure, J. Stat. Phys. 175 (2019) 987 [arXiv: 1707.00965].

[9] A. Gamsa and J. Cardy, Correlation functions of twist operators applied to single self-avoiding loops, J. Phys. A 39 (2006) 12983.

[10] D. Beliaev and F.J. Viklund, Some remarks on SLE bubbles and Schramm's two-point observable, Commun. Math. Phys. 320 (2013) 379 [arXiv:1012.5206].

[11] B. Freivogel and M. Kleban, A conformal field theory for eternal inflation, JHEP 12 (2009) 019 [arXiv: 0903.2048] [INSPIRE].

[12] B. Freivogel, M. Kleban, A. Nicolis and K. Sigurdson, Eternal inflation, bubble collisions and the disintegration of the persistence of memory, JCAP 08 (2009) 036 [arXiv:0901.0007] [INSPIRE].

[13] Y. Ikhlef, J.L. Jacobsen and H. Saleur, Three-point functions in $c \leq 1$ Liouville theory and conformal loop ensembles, Phys. Rev. Lett. 116 (2016) 130601 [arXiv:1509.03538] [INSPIRE].

[14] H. Dorn and H.J. Otto, On correlation functions for noncritical strings with $c \leq 1 d \geq 1$, Phys. Lett. B 291 (1992) 39 [hep-th/9206053] [InSPIRE].

[15] A.B. Zamolodchikov and A.B. Zamolodchikov, Structure constants and conformal bootstrap in Liouville field theory, Nucl. Phys. B 477 (1996) 577 [hep-th/9506136] [InSPIRE].

[16] M. Picco, S. Ribault and R. Santachiara, A conformal bootstrap approach to critical percolation in two dimensions, SciPost Phys. 1 (2016) 009 [arXiv:1607.07224] [INSPIRE].

[17] J. Lykke Jacobsen and H. Saleur, Bootstrap approach to geometrical four-point functions in the two-dimensional critical Q-state Potts model: a study of the s-channel spectra, JHEP 01 (2019) 084 [arXiv: 1809.02191] [INSPIRE].

[18] F. Camia, A. Gandolfi, G. Peccati and T.R. Reddy, Brownian loops, layering fields and imaginary Gaussian multiplicative chaos, arXiv:1908.05881 [INSPIRE].

[19] P. Di Francesco, P. Mathieu and D. Sénéchal, Conformal field theory, Springer-Verlag, New York, NY, U.S.A. (1997) [INSPIRE].

[20] M. Headrick, Mathematica packages webpage, http://people.brandeis.edu/ headrick/Mathematica/index.html.

[21] J.J.H. Simmons and J. Cardy, Twist operator correlation functions in $O(n)$ loop models, J. Phys. A 42 (2009) 235001.

[22] S. Nacu and W. Werner, Random soups, carpets and fractal dimensions, J. London Math. Soc. 83 (2011) 789. 